\title{
miR-511-3p protects against cockroach allergen-induced lung inflammation by antagonizing CCL2
}

\author{
Danh C. Do, ${ }^{1}$ Jie Mu, ${ }^{1,2}$ Xia Ke, ${ }^{3}$ Karan Sachdeva, ${ }^{1}$ Zili Qin, ${ }^{4}$ Mei Wan, ${ }^{5}$ Faoud T. Ishmael, ${ }^{6}$ \\ and Peisong Gao'
}

Johns Hopkins Asthma and Allergy Center, Johns Hopkins University School of Medicine, Baltimore, Maryland, USA. 2Department of Anesthesiology, West China School of Medicine, Sichuan University, Chengdu, China. ${ }^{3}$ Department of Otorhinolaryngology, First Affiliated Hospital of Chongqing Medical University, Chongqing, China. ${ }^{4}$ Otorhinolaryngology Hospital, The First Affiliated Hospital, Sun Yat-sen University, Guangzhou, China. ${ }^{5}$ Department of Orthopedic Surgery, Johns Hopkins University School of Medicine, Baltimore, Maryland, USA. 'Department of Medicine, Division of Pulmonary, Allergy, and Critical Care Medicine, Pennsylvania State University Milton S. Hershey Medical Center, Hershey, Pennsylvania, USA.

miR-511-3p, encoded by CD206/Mrc1, was demonstrated to reduce allergic inflammation and promote alternative (M2) macrophage polarization. Here, we sought to elucidate the fundamental mechanism by which miR-511-3p attenuates allergic inflammation and promotes macrophage polarization. Compared with WT mice, the allergen-challenged $\mathrm{MrC1}^{-1-}$ mice showed increased airway hyperresponsiveness (AHR) and inflammation. However, this increased AHR and inflammation were significantly attenuated when these mice were pretransduced with adenoassociated virus-miR-511-3p (AAV-miR-511-3p). Cene expression profiling of macrophages identified $C c / 2$ as one of the major genes that was highly expressed in M2 macrophages but antagonized by miR-511-3p. The interaction between miR-511-3p and $C c / 2$ was confirmed by in silico analysis and mRNA-miR pulldown assay. Further evidence for the inhibition of $C c / 2$ by miR-511-3p was given by reduced levels of Ccl2 in supernatants of miR-511-3p-transduced macrophages and in bronchoalveolar lavage fluids of AAV-miR-511-3p-infected $\mathrm{Mrc1}^{-1-}$ mice. Mechanistically, we demonstrated that Ccl2 promotes M1 macrophage polarization by activating RhoA signaling through Ccr2. The interaction between Ccr2 and RhoA was also supported by coimmunoprecipitation assay. Importantly, inhibition of RhoA signaling suppressed cockroach allergen-induced AHR and lung inflammation. These findings suggest a potentially novel mechanism by which miR-511-3p regulates allergic inflammation and macrophage polarization by targeting $\mathrm{Ccl} 2$ and its downstream Ccr2/RhoA axis.

Authorship note: DCD and JM contributed equally to this work.

Conflict of interest: The authors have declared that no conflict of interest exists.

Copyright: (c) 2019, American Society for Clinical Investigation.

Submitted: December 14, 2018 Accepted: September 12, 2019 Published: September १९, 2019.

Reference information: JCl Insight. 2019;4(20):e126832. https://doi.org/10.1172/jci. insight.126832.

\section{Introduction}

C-type lectin receptors (CLRs) are pattern-recognition receptors that recognize allergenic glycans present on allergens, facilitating the endocytosis and presentation of pathogens that are critical in shaping the development of allergic asthma (1-8). Most importantly, macrophage mannose receptor (MRC1/CD206), one of the major CLRs, has been shown to play an important role in mediating allergic sensitization (9, 10), lung inflammation (10), and activation of innate immune cells $(4,11)$. Our previous studies identified a critical role of MRC1 in allergen clearance as a natural defense mechanism and in limiting the progression and severity of allergic inflammation induced by cockroach allergen (cockroach extract, CRE) in a mouse model of asthma (10). In particular, Mrcl-deficient mice showed significant reduction in CRE uptake, but unexpectedly, these mice have an exacerbation of CRE-induced airway inflammation. Although the underlying mechanisms remain uncertain, our studies suggest that $M r c 1$ deficiency in macrophages may lead to the alterations in allergen uptake and balance between classically activated (or M1) and alternatively activated (or M2) macrophage phenotypes (10) that may contribute to the exacerbation of airway inflammation.

To further explore the underlying mechanisms, we have focused on miR-511-3p, encoded by CD206/Mrc1. microRNAs (miRs) are small noncoding RNAs that regulate gene expression and cell func- 
tion by affecting the translation and stability of their targeted mRNAs (12). miRs can target genes that orchestrate T helper 2 responses $(13,14)$, phagocyte activation $(10,15)$, and shape macrophage polarization and plasticity $(16,17)$, all of which are important immunological processes in the pathogenesis of diseases (18). miR-511-3p is transcriptionally coregulated with the MRC1 gene in macrophages $(10,19)$. Recent studies have suggested that miR-511-3p controls the activation of tumor-associated macrophages (19), regulates intestinal inflammation by targeting Toll-like receptor 4 (TLR4) (20), and affects dendritic cell (DC) function by cross-talk with CLRs (21). Profiling of macrophages demonstrated opposing expression patterns for miR-511 in M1 and M2 macrophages, with an increased expression of miR-511$3 p$ in M2, but decreased expression in M1 macrophages (22). Our previous studies have also suggested a role for miR-511-3p, the functional strand of miR-511, in shaping the balance between M1 and M2 macrophage polarization (10). Furthermore, by using WT mice, we showed that miR-511-3p protects against allergen-induced airway inflammation. However, the underlying mechanisms by which miR-511$3 p$ modulates macrophage polarization and lung inflammation have not yet been fully elucidated.

In the current study, we have provided evidence that miR-511-3p can inhibit the increased CREinduced airway hyperresponsiveness (AHR) and lung inflammation caused by the lack of Mrc1 in mice. We have also attempted to elucidate the underlying mechanism. Specifically, we focused on $C c l 2$, one of the major miR-511-3p target genes identified by gene array, which showed an increased expression in M2 macrophages but was antagonized by miR-511-3p. Finally, we explored the role of the Ccl2 pathway in macrophage polarization and allergic airway inflammation.

\section{Results}

Transfection of miR-511-3p in mice attenuates CRE-induced AHR and lung inflammation caused by Mrc1 deficiency. We have previously demonstrated an exacerbation of CRE-induced lung inflammation in $\mathrm{Mrcl}^{-/-}$mice when compared with WT mice (10). To determine whether the increased inflammation in $\mathrm{Mrcl}^{{ }^{\prime-}}$ mice is due to the lack of the Mrcl-encoded miR-511-3p, we generated an adeno-associated virus-GFP (AAVGFP) vector carrying miR-511-3p to transduce $\mathrm{Mrcl}^{-1-}$ mice according to the experimental approach that we have previously reported (10). We first checked major lung cell populations that were transduced by AAV-miR-511-3p using flow cytometry analysis and coimmunostaining. Among the $\mathrm{GFP}^{+}$cells, $42.1 \%$ were macrophages $\left(\mathrm{F} 4 / 80^{+} \mathrm{CD} 11 \mathrm{c}^{+}\right), 23.1 \%$ were DCs $\left(\mathrm{CD} 11 \mathrm{c}^{+} \mathrm{MHCII}{ }^{\mathrm{hi}}\right)$, and $26.8 \%$ were epithelial cells $\left(\mathrm{MHCII}^{\mathrm{hi}} \mathrm{EpCaM}^{+}\right)$(Supplemental Figure 1A; supplemental material available online with this article; https://doi.org/10.1172/jci.insight.126832DS1). These transduced macrophages and airway epithelial cells were further confirmed by GFP coimmunostaining with either F4/80 (macrophages) or EpCaM (epithelial cells) (Supplemental Figure 1B). Increased miR-511-3p was also noted in the lung tissues of these transduced mice as assessed by RT-PCR (Supplemental Figure 1C). We then used these transduced mice to generate a mouse model, as illustrated in Figure 1A. Compared with WT mice, CRE-treated $\mathrm{Mrcl}^{-/-}$mice showed an increased airway resistance, which was reduced when these $\mathrm{Mrcl}^{-1-}$ mice were transduced with AAV-miR-511-3p (Figure 1B). Moreover, consistent with our previous findings (10), CRE-treated $\mathrm{MrCl}^{-1}$ mice had exacerbated lung inflammation when compared with WT mice (Figure 1, C-F). Interestingly, the increased lung inflammation was remarkably attenuated when these $\mathrm{Mrcl}^{-/-}$mice were transduced with AAV-miR-511-3p in comparison with AAV-control. Particularly, Mrc1 ${ }^{-/}$mice with AAV-miR-511-3p treatment showed a significant reduction in the recruitment of inflammatory cells to the lung, with dense peribronchial infiltrates and goblet cell hyperplasia in the histological examination (Figure 1C). Compared with $\mathrm{Mrcl}^{-/-}$mice, these AAV-miR-511-3p-treated $\mathrm{Mrcl}^{\mathrm{I}^{-1}}$ mice displayed a much lower number of total inflammatory cells, especially eosinophils and neutrophils, in the bronchoalveolar lavage (BAL) fluids (Figure 1D). Furthermore, these miR-511-3p-treated $\mathrm{MrCl}^{-/-}$mice showed reduced serum levels of CRE-specific IgE (sIgE) and IgG1 (sIgG1) (Figure 1E), lower levels of IL-4, IL-13, and IL-17, but higher levels of IFN- $\gamma$ and IL-10 in the BAL fluids (Figure 1F). Collectively, our studies suggest that miR-511-3p treatment can block the increased AHR and allergic lung inflammation caused by Mrcl deficiency.

miR-511-3 polarizes Mrc1-deficient macrophages into M2 phenotypes. We have previously demonstrated that MRC1 regulates macrophage polarization through miR-511-3p (10). To further support this finding, we analyzed the M1 and M2 macrophages in CRE-treated $\mathrm{MrCl}^{-{ }^{--}}$mice with or without AAV-miR-511-3p transduction (Figure 2A). Lung tissue M1 and M2 macrophages were assessed by coimmunostaining for F4/80 with either iNOS (M1) or Arg-1 (M2). Of these lung macrophages (F4/80+), Mrc1 ${ }^{-/-}$mice treated with AAV-miR-511-3p showed decreased M1 macrophages (F4/80 ${ }^{+}$NOS $^{+}$cells, Figure 2, A and B), but 
A
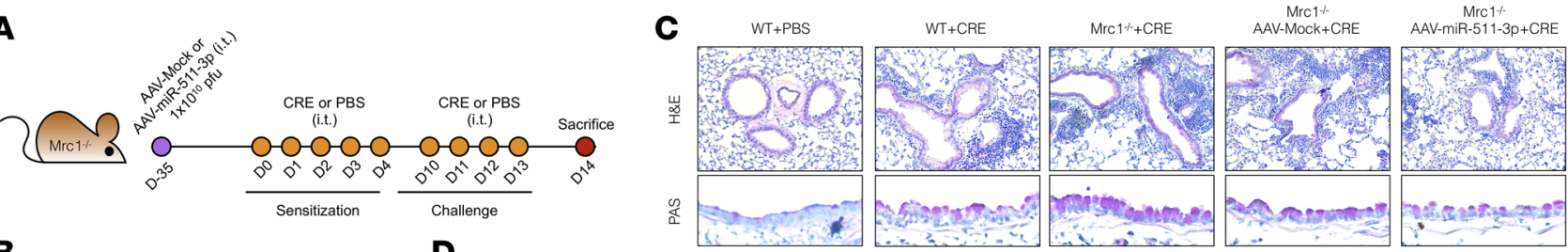

B

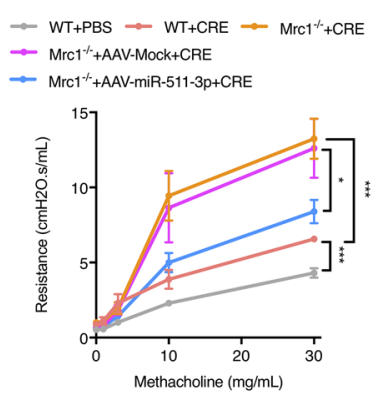

\section{D}
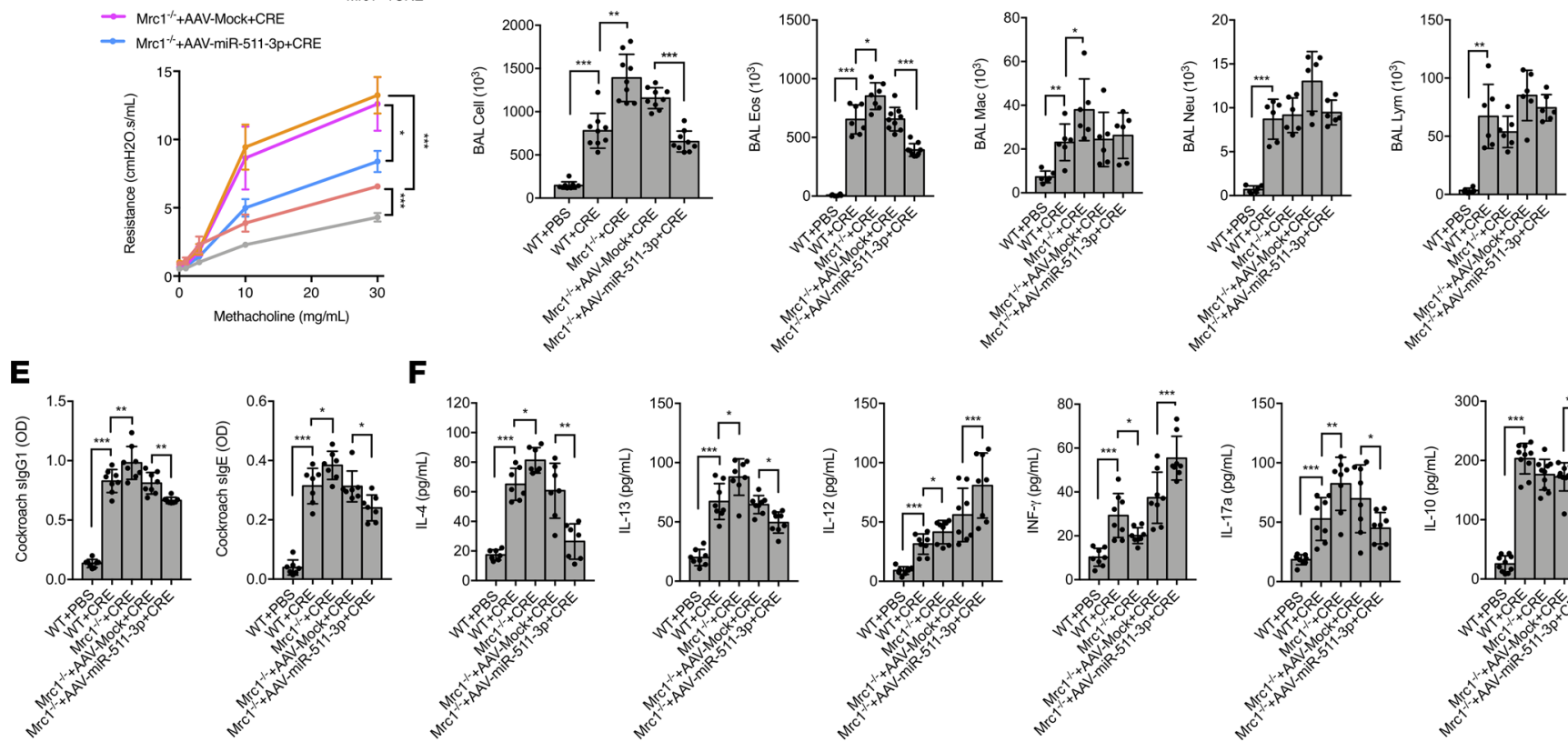

$\mathbf{F}$
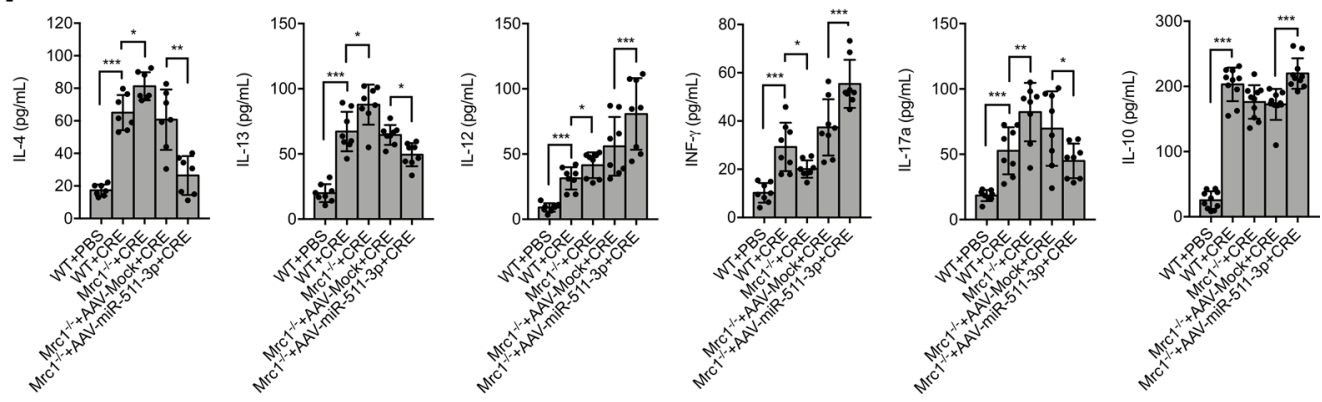

Figure 1. Transfection of miR-511-3p blocks cockroach allergen-induced airway hyperresponsiveness and lung inflammation caused by mouse Mrc1 deficiency. (A) Protocol for AAV-mediated transduction of miR-511-3p into $\mathrm{Mrc1}^{-1-}$ mice and cockroach allergen-induced mouse model of asthma. (B) Lung resistance in response to increasing concentrations of methacholine using the forced oscillation technique (FlexiVent, SCIREQ) ( $n=5 / g r o u p)$. (C) Histological examination of mouse paraffin lung sections stained with hematoxylin and eosin (H\&E, upper panel) and periodic acid-Schiff (PAS, lower panel). (D) Bronchoalveolar lavage (BAL) fluid total and differential (eosinophil, macrophage, neutrophil, and lymphocyte) cell counts as assessed by flow cytometry ( $n=8-9 / g r o u p)$. (E) Serum levels of cockroach allergen-specific IgE and IgG1 ( $n=6 / g r o u p)$. (F) BAL fluid levels of cytokines ( $n=6 / g r o u p)$. Data in B and D-F were compared by 2-way ANOVA. ${ }^{*} P<0.05 ;{ }^{* *} P<0.01 ;{ }^{* * *} P<0.001$.

increased M2 macrophages (F4/80+Arg-1+ cells, Figure 2, A and C), when compared with AAV-control. To further characterize lung macrophages from these in vivo-challenged mice, we performed flow cytometry with additional markers to characterize the lung M1/M2 macrophages of AAV-miR-511-3p-treated and AAV-control-treated mice (Figure 2D). Specifically, we gated on M2 macrophages with markers F4/80, $\mathrm{CD} 11 \mathrm{c}$, and Arg-1 (F4/80 $\mathrm{CD} 11 \mathrm{c}^{+} \mathrm{Arg}-1^{+}$cells) and on M1 macrophages with markers F4/80, CD11c, and iNOS (F4/80 ${ }^{+} \mathrm{CD}_{11} \mathrm{c}^{+} \mathrm{iNOS}^{+}$cells). Consistent with coimmunostaining, AAV-miR-511-3p-treated mice showed fewer M1 (F4/80 ${ }^{+} \mathrm{CD} 11 \mathrm{c}^{+} \mathrm{NNOS}^{+}$cells), but more M2 macrophages (F4/80 $0^{+} \mathrm{CD} 11 \mathrm{c}^{+} \mathrm{Arg}-1^{+}$ cells) (Figure 2E). These findings were supported by the analyses of macrophages (Supplemental Figure 3) enriched from BAL fluids of CRE-challenged $\mathrm{Mrcl}^{-/-}$mice with or without AAV-miR-511-3p treatment (Figure 2F). AAV-miR-511-3p-treated mice have reduced M1, but increased M2 macrophages. Importantly, we detected the expression of M1- and M2-associated genes in these BAL macrophages. As expected, macrophages from AAV-511-3p-treated mice showed decreased expression of M1-associated genes (IL-1 1 , IL-6, and iNOS), but increased expression of M2-associated genes (Arg-1, Fizz1, and Chi3l3) (Figure $2 \mathrm{G})$. These findings were also supported by in vitro analyses with bone marrow-derived macrophages (BMDMs). BMDMs from $\mathrm{Mrc1}^{-/-}$mice were transfected with lentivirus-miR-511-3p (LV-miR-511-3p) or LV-mock control, and then cultured under either M1 (LPS) or M2 (IL-4) conditions. Compared with LV-mock, LV-miR-511-3p-infected BMDMs showed reduced expression of M1-associated genes (IL-1 $I L-6$, and iNOS), but increased expression of M2-associated genes (Arg-1, Fizz1, and Chi3l3) (Figure 2H). Taken together, these results support our previous findings and further suggest that miR-511-3p polarizes macrophages toward the M2 phenotype independent of Mrc1 expression. 
A

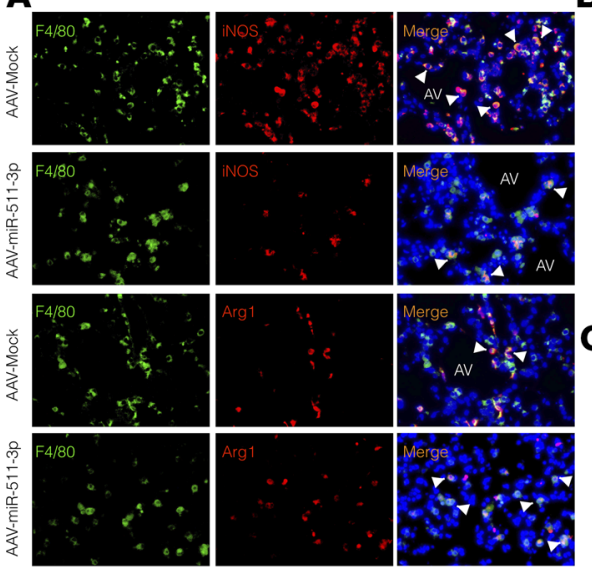

B

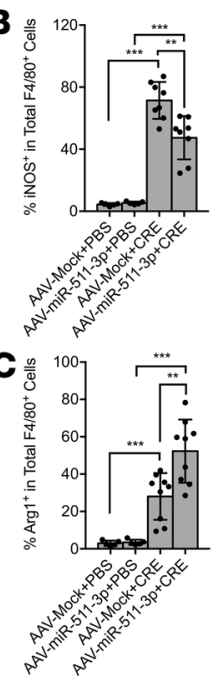

D
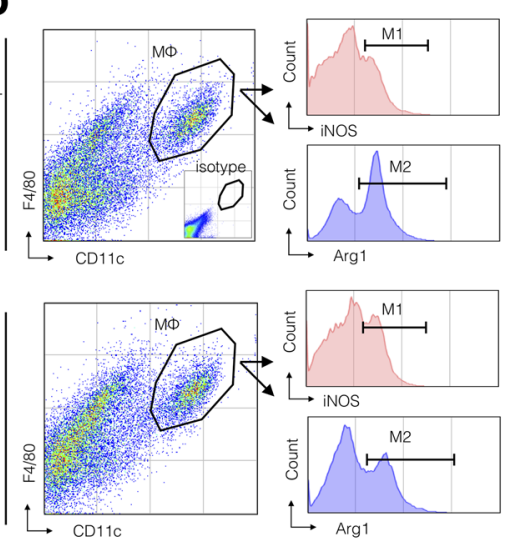

$\mathbf{E}$
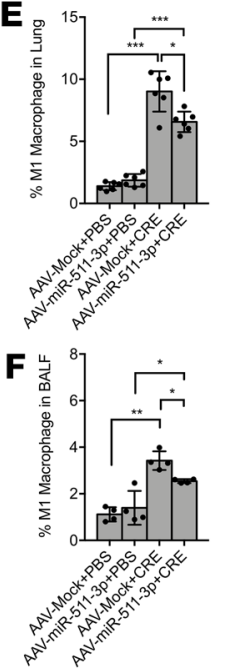
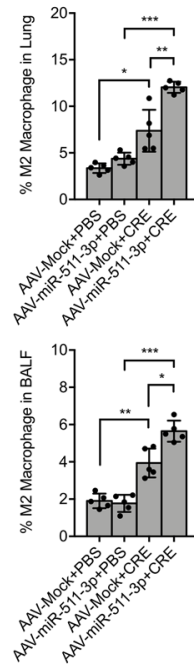

G

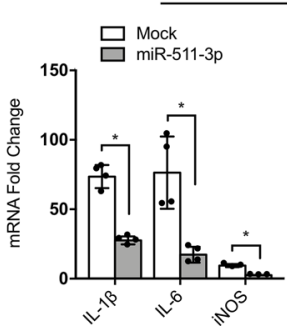

$\operatorname{Mrc1-:M\Phi }$

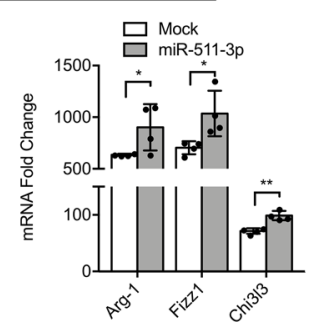

H

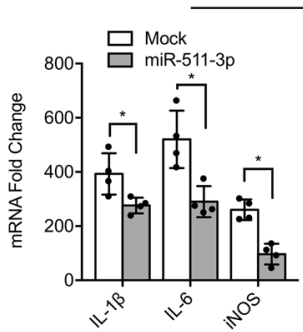

Mrc1- :BMDM

Figure 2. miR-511-3p polarizes Mrc1-deficient macrophages into M2 phenotypes. (A) Representative images of double-immunofluorescence staining with primary antibodies against F4/80, iNOS, and Arg-1. Nuclei were counterstained with 4'-6-diamidino-2-phenylindole dihydrochloride (DAPI). Original magnification, $\times 20$. (B and C) Quantitation of lung tissue M1 and M2 macrophages as indicated by the percentage of F4/80+iNOS+ (B, \%M1) and F4/80 $0^{+}$rg- $1^{+}\left(\mathbf{C}, \% \mathrm{M}^{+}\right)$ among total F4/80+ cells, respectively ( $n=8-9$ high power view/group). (D) Gating strategies for the flow cytometry analysis of M1/M2 macrophages in the lung tissues and BAL fluids of mice treated with AAV-miR-511-3p (top panel) and AAV-control (bottom panel). (E and F) Quantitation of M1 and M2 macrophages as indicated by the percentage of $\mathrm{F} 4 / 80^{+} \mathrm{CD} 11 \mathrm{c}^{+} \mathrm{iNOS}{ }^{+}$cells (\%M1) and $\mathrm{F} 4 / 80^{+} \mathrm{CD} 11 \mathrm{c}^{+} \mathrm{Arg}-\mathrm{1}^{+}$cells $(\% \mathrm{M} 2)$ among total lung single cells from lung tissues (E) and BAL fluids (F) ( $n=5$ /group). ( $\mathbf{G}$ and $\mathbf{H})$ Quantitative RT-PCR analyses for M1- and M2-associated genes in macrophages enriched from BAL fluids ( $n=4$ / group) and bone marrow-derived macrophages (BMDMs) ( $n=4$ /group) with lentiviral vector-miR-511-3p (LV-miR-511-3p) or LV-Mock (empty vector) transfection. Data in B, C, E, and $\mathbf{F}$ were compared by 2-way ANOVA. Data in $\mathbf{G}$ and $\mathbf{H}$ were compared by 2-tailed Student's $t$ test. ${ }^{*} P<0.05 ;{ }^{* *} P<0.01 ;{ }^{* *} P<0.001$.

$C c 12$ is one of the miR-511-3p target genes. To explore the underlying mechanisms by which miR-511-3p polarizes macrophages toward the M2 phenotype, we performed transcriptional profiling of undifferentiated macrophages, M2 macrophages (under M2 culture conditions with IL-4), and M2 macrophages pretreated with an miR-511-3p mimic using the MouseRef-8 v2.0 BeadChip, as previously described (10). From gene array analysis, we prioritized the 10 most differentially expressed genes that are increased in M2 macrophages as compared to the undifferentiated macrophages, but were significantly decreased by the miR-511-3p mimic (Figure 3A). We then narrowed down the number of genes based on their potential association with macrophage polarization and asthma and further confirmed their expression by qRT-PCR. These genes include $\mathrm{Ccl} 2$ (chemokine ligand 2) (23, 24), Ak2 (adenylate kinase 2) (25), Clcn3 (chloride voltage-gated channel 3) (26), Plek (pleckstrin) (27), and Srgn (serglycin) (ref. 28 and Figure 3B). Consistent with the gene array analysis, these selected genes are highly expressed in M2 macrophages as compared with undifferentiated macrophages, but significantly inhibited when these M2 macrophages were pretreated with the miR-511-3p mimic, with the exception of $A k 2$. Furthermore, we performed in silico analysis by using BiBiServ RNAhybrid, a program that predicts multiple potential binding sites of miRs in large target RNAs (29). The predicted binding sites for miR-511-3p in the 3'-UTR for these genes are provided in Supplemental Table 1. In particular, we predicted the miR-511-3p binding site in human CCL2 mRNAs (+93-111, Figure 4A) and murine Ccl2 (+94-111, Figure 4B). The miR-511-3p-Cc12 binding was further supported by the miR-mRNA pulldown assay, as outlined in Figure 4C. Compared with miR control, a significant amount of Ccl2 mRNA was detected in miR-511-3p-biotin-mRNA complexes from IL-4-treated BMDMs (Figure 4D). Furthermore, LV-miR-511-3p, but not LV-control, transduction significantly inhibited $\mathrm{Ccl} 2$ secretion as assessed by the levels of $\mathrm{Ccl} 2$ in supernatants of IL-4-treated BMDMs (M2 macrophages, Fig- 

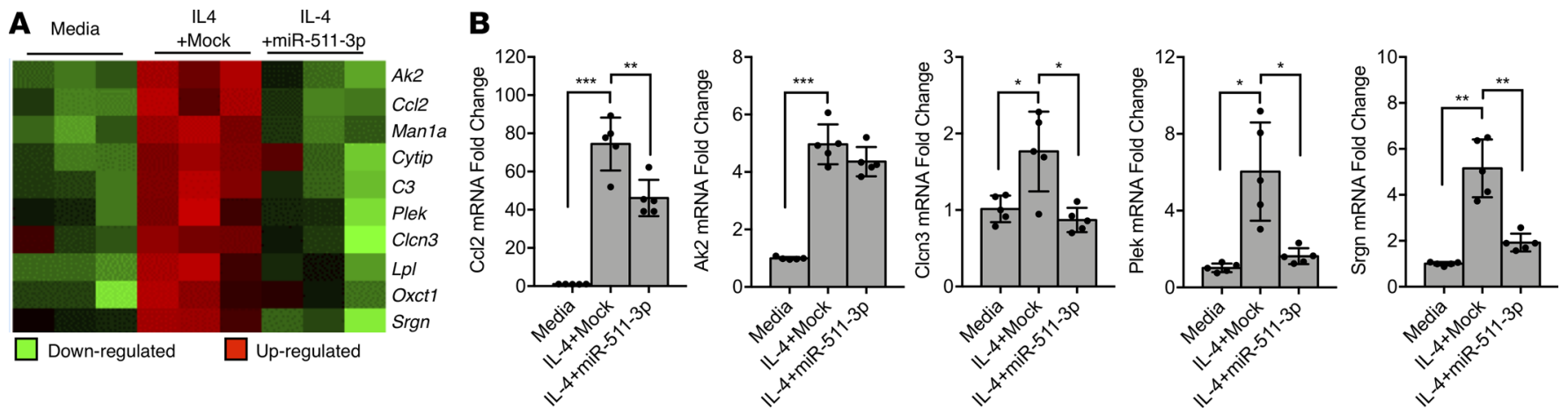

Figure 3. Identification of miR-511-3p target genes by gene array. (A) Gene expression profiling of undifferentiated macrophages, M2 macrophages, and M2 macrophages with miR-511-3p mimic pretreatment using the MouseRef-8 v2.0 BeadChip. (B) Quantitative RT-PCR analyses for several selected genes. $n=5$ /group. Comparisons were made by 2 -way ANOVA. ${ }^{*} P<0.05$; ${ }^{* *} P<0.01 ;{ }^{* *} P<0.001$.

ure 4E). Additionally, we analyzed the levels of Ccl2 in BAL fluids of miR-511-3p-treated mice. Compared with WT mice, Ccl2 levels were increased in BAL fluids from CRE-challenged $\mathrm{Mrcl}^{---}$mice, but significantly reduced when these mice were preinfected with AAV-miR-511-3p (Figure 4F). Taken together, these findings suggest that $\mathrm{Ccl} 2$ is one of the miR-511-3p targets that may contribute to the increased risk of asthma.

Ccl2 promotes $M 1$ macrophage polarization through $C c r 2$. As a miR-511-3p target gene, we investigated whether Ccl2 plays a role in miR-511-3p-regulated macrophage polarization. Similarly to LPS, when BMDMs were directly treated with $\mathrm{Ccl} 2$, these cells showed increased expression of the M1-associated marker iNOS, as detected by immunostaining (Figure 5, A and B). In contrast, Cc12-treated BMDMs showed no change for Arg-1, an M2-associated marker that was significantly expressed in IL-4-treated BMDMs (Figure 5, A and C). These findings were further supported by qRT-PCR. Cc12-treated BMDMs showed significantly increased expression of M1-associated genes ( $I L-1 \beta$, IL-6, and $i N O S$, Figure 5D), but no change was noted for M2-associated genes (Arg-1, Fizz1, and Chi3l3, Figure 5E). Next, we investigated the molecular mechanisms by which $\mathrm{Ccl} 2$ induces macrophage polarization through its receptor $\mathrm{Ccr} 2$. Ccr2 was knocked down in BMDMs by Ccr2 siRNA, but not control siRNA, as assessed by qRT-PCR (Figure 5F). These BMDMs with or without $\mathrm{C} c 2$ knockdown were then treated with $\mathrm{Cc1} 2$ for the analysis of macrophage polarization. As expected, BMDMs with $\mathrm{C} c r 2$ siRNA knockdown showed reduction in the expression of M1-associated genes (Figure $5 \mathrm{G}$ ), but no changes for M2-associated genes (Figure $5 \mathrm{H}$ ), as compared with those with control siRNA. These results indicate that $\mathrm{Ccr} 2$ is essential in Cc12-promoted $\mathrm{M} 1$ macrophage polarization.

Ccl2 activates RhoA signaling that contributes to macrophage polarization. We have recently reported that RhoA signaling is critical in CRE-induced airway inflammation/remodeling and mesenchymal stem cell differentiation in a chronic mouse model of asthma (30). Thus, we investigated whether RhoA signaling also plays a role in Cc12-induced macrophage differentiation. First, BMDMs were treated with Cc12; RhoA activity was investigated by immunostaining for RhoA-GTP. Similarly to LPS-treated BMDMs, Ccl2 induced increased expression of RhoA-GTP in BMDMs (Figure 6A). Next, we used fasudil, a selective RhoA/ROCK inhibitor (31), to explore whether RhoA signaling can regulate macrophage polarization. Compared with untreated BMDMs, Ccl2-treated BMDMs expressed higher levels of the M1-associated genes $I L-1 \beta, I L-6$, and iNOS (Figure 6B). These increased M1 genes were inhibited when BMDMs were pretreated with fasudil for 30 minutes prior to Cc12 stimulation, suggesting that RhoA signaling is crucial for Ccl2-induced M1 macrophage polarization. To further confirm the role of RhoA in macrophage polarization, active RhoA overexpression in BMDMs was generated by transfecting plasmids containing a constitutively active RhoA (RhoA-L63), and then treated with Cc12. Consistent with the previous findings, RhoA-L63-transfected BMDMs showed increased expression of M1-associated genes when compared with those transfected with RhoA-WT (Figure 6C). Lastly, we explored whether there is a physiological protein-protein interaction between CCR2 and RhoA in macrophages by using coimmunoprecipitation assay. Both CCR2 and RhoA are expressed in the human monocyte-derived cell line THP-1 (ref. 32 and Figure 6D, Input). Although RhoA expression in these samples is much lower in comparison with CCR2, a complex of CCR2 coprecipitated with RhoA was identified by Western blot, suggesting that CCR2 could interact with RhoA. Together with the data on the role of RhoA signaling in macrophage polarization, these results show that $\mathrm{Ccl} 2$ induces macrophage polarization through the $\mathrm{Ccr} 2 / \mathrm{RhoA}$ axis. 
A

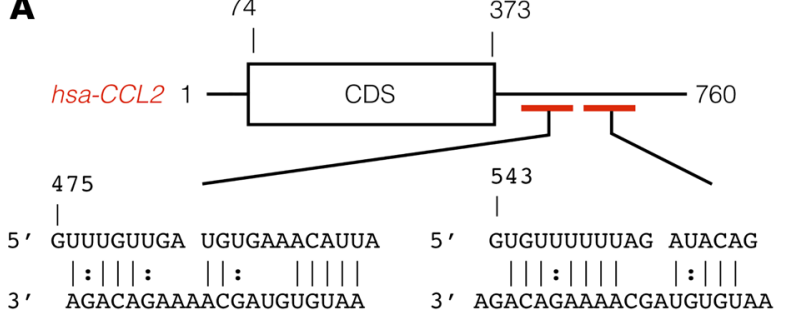

B

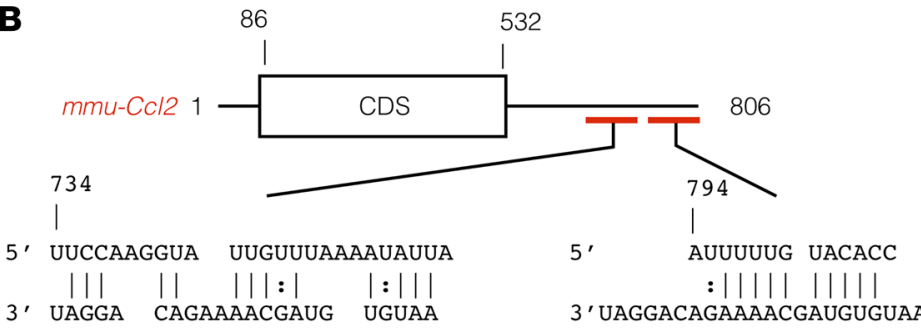

C

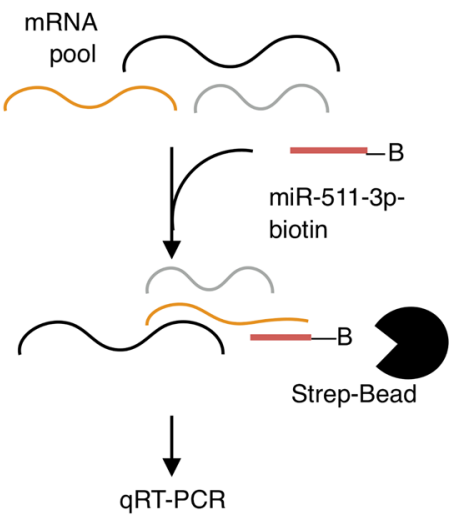

D

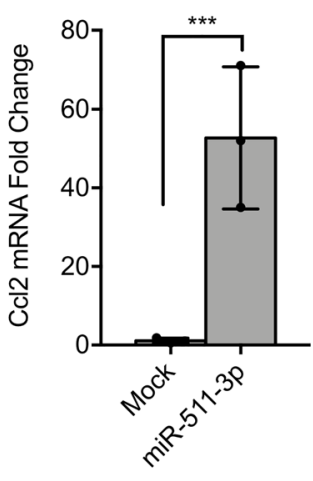

E

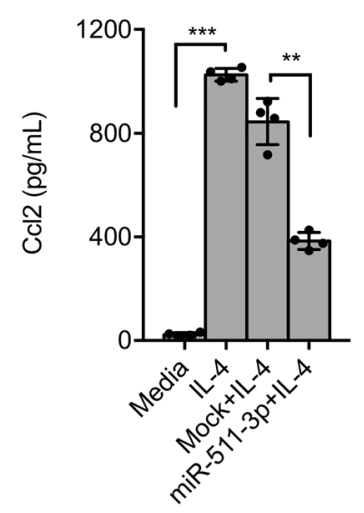

$\mathbf{F}$

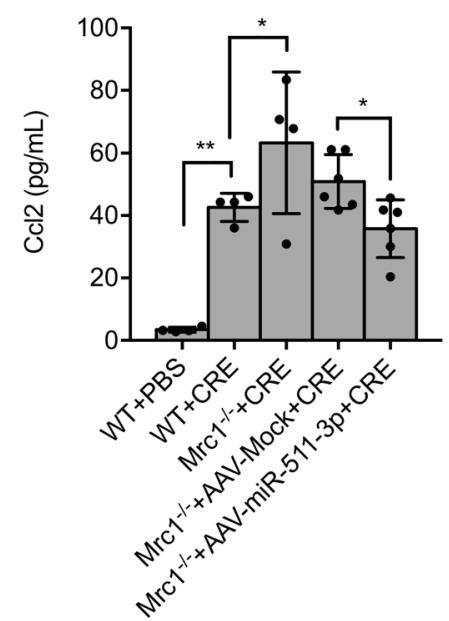

Figure 4. Ccl2 is one of the miR-511-3p target genes. (A and B) In silico prediction of miR-511-3p binding site in Ccl2 mRNA in human (hsa-CCL2, A) and mouse (mmu-Ccl2, B) by BiBiServ RNAhybrid. (C) Schematic representation of miR-511-3p-mRNA biotin pulldown assay. B, biotin. (D) Quantitative RT-PCR analyses of Cc/2 in miR-511-3p-mRNA complexes that were isolated from LV-miR-511-3p or LV-Mock control-transfected macrophages ( $n=3 /$ group). ( $\mathbf{E}$ and $\mathbf{F}$ ) ELISA analysis of CcI2 levels in supernatants of cultured LV-miR-511-3p or LV-Mock-transfected BMDMs ( $E, n=4 /$ group) and mouse BAL fluids of AAV-miR-511-3p-infected $\mathrm{Mrc1}^{-1-}$ mice ( $\mathbf{F}, n=6 /$ group). Data in $\mathbf{D}$ were compared by 2-tailed Student's $t$ test. Data in $\mathbf{E}$ and $\mathbf{F}$ were compared by 2-way ANOVA. ${ }^{*} P<0.05 ;{ }^{*} P<0.01 ;{ }^{* *} P<0.001$.

RhoA signaling regulates lung inflammation in a mouse model. Given the role of RhoA signaling in mediating Cc12-promoted macrophage polarization, we further investigated whether the Ccl2-activated RhoA signaling can affect allergen-induced AHR and lung inflammation in our mouse model of acute asthma, as reported previously (33). In particular, we examined whether inhibition of RhoA signaling can prevent CRE-induced AHR and lung inflammation by using fasudil before CRE challenge, following the protocol illustrated in Figure $1 \mathrm{~A}$. Consistent with our previous findings $(30,33), \mathrm{CRE}$ induced increased airway resistance (Figure 7A) and airway inflammation (Figure 7, B-E) when compared with PBS treatment. Of interest, when these allergen-challenged mice were treated with fasudil, the increased airway resistance and lung inflammation were significantly attenuated. Specifically, treatment with fasudil significantly suppressed CRE-induced peribronchial inflammation and goblet cell hyperplasia (Figure 7B). Furthermore, these fasudil-treated mice showed a significant reduction in the number of total inflammatory cells, eosinophils, macrophages, neutrophils, and lymphocytes in the BAL fluids (Figure 7C). Similarly, we found that serum levels of CRE-induced sIgE and sIgG1 were also remarkably reduced in these fasudil-treated mice (Figure 7D). The same pattern was noted for the levels of IL-4, IL-5, IL-13, IFN- $\gamma$, and IL-17 $\alpha$ (Figure 7E). However, no changes were noted for IL-12 and IL-10. These results provide evidence that RhoA signaling is critical in CRE-induced AHR and lung inflammation.

\section{Discussion}

The goal of our study was to explore the role of miR-511-3p and the fundamental mechanism by which miR-511-3p plays a role in Mrc1-mediated CRE-induced lung inflammation. We have previously demonstrated that $\mathrm{MrCl}^{-1-}$ mice showed an exacerbation of CRE-induced lung inflammation (10). In this study, we have provided evidence that the increased AHR and lung inflammation due to the lack of Mrcl can be reversed by AAV-mediated transduction of miR-511-3p. Furthermore, by gene array analysis, we have iden- 
A

Media

$\mathrm{Ccl} 2$

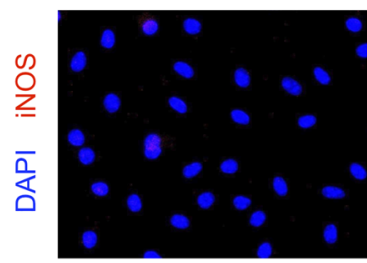

Media
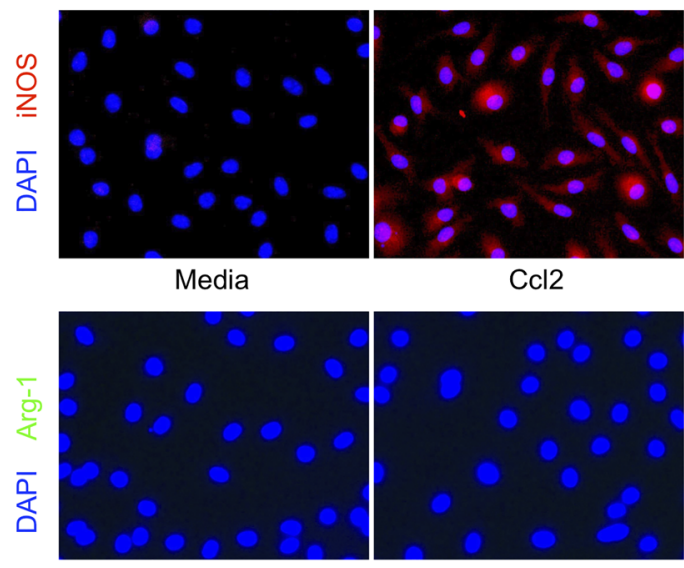

Ccl2
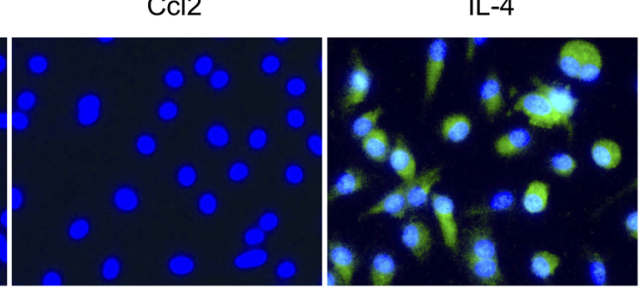

B

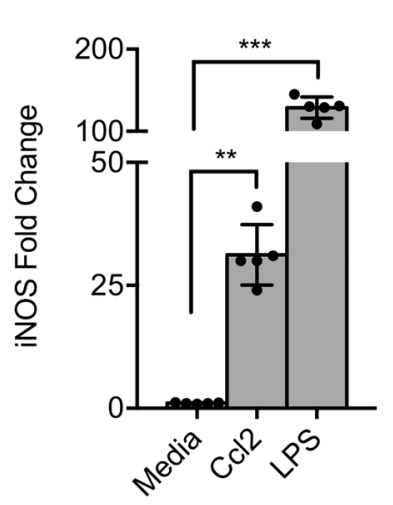

C

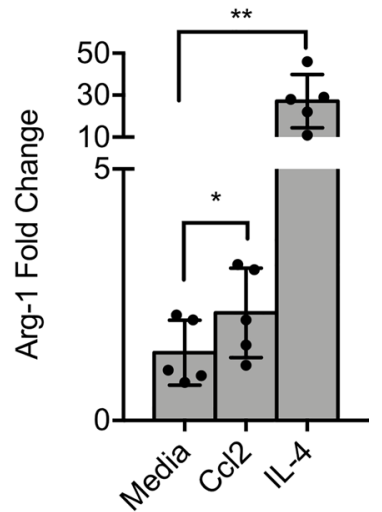

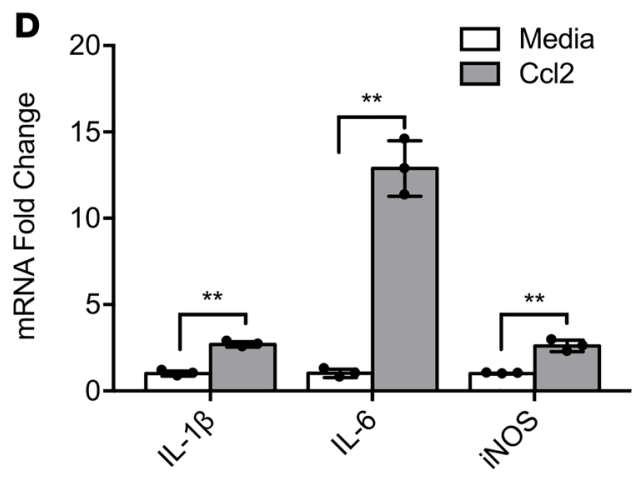

E
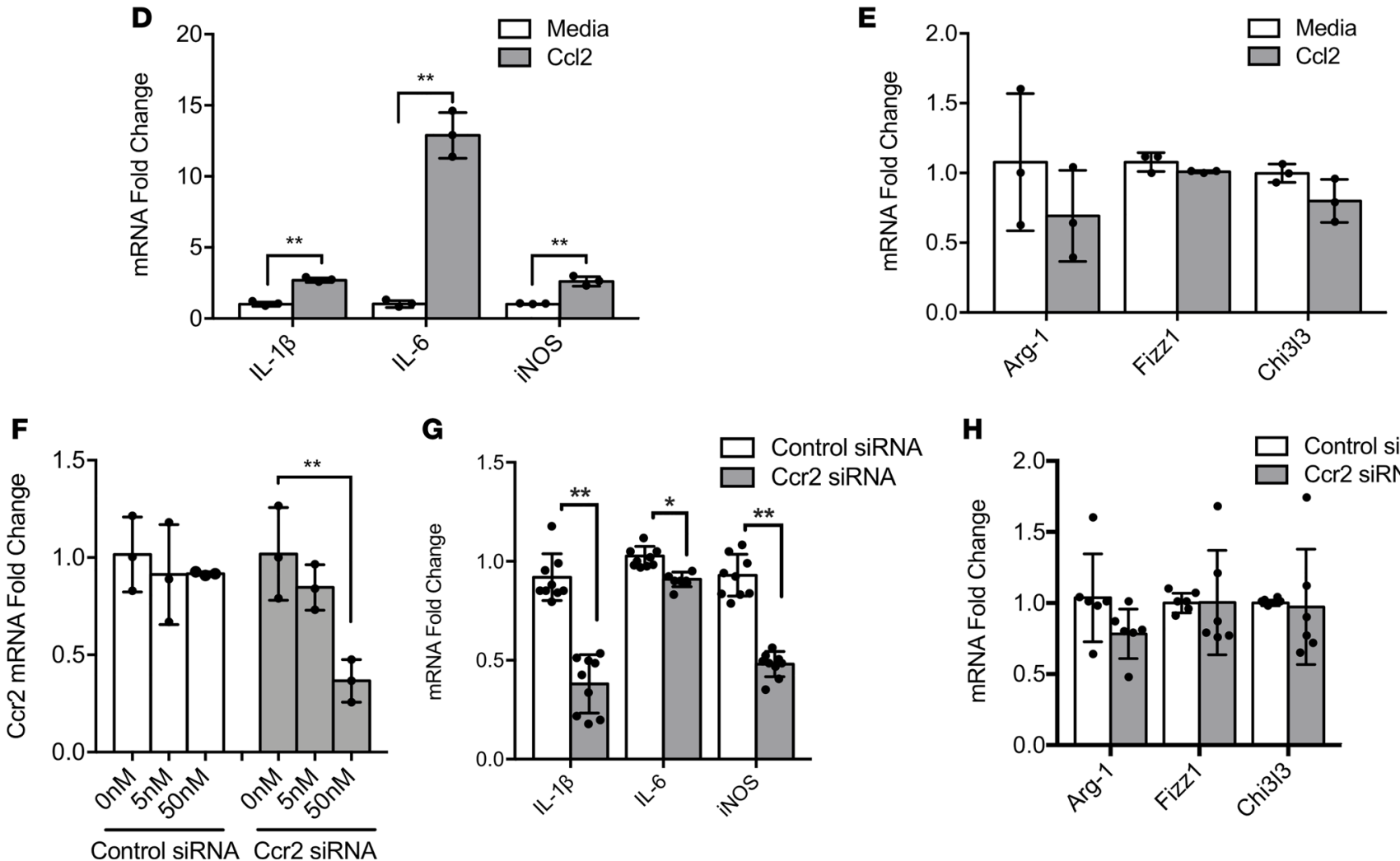

G
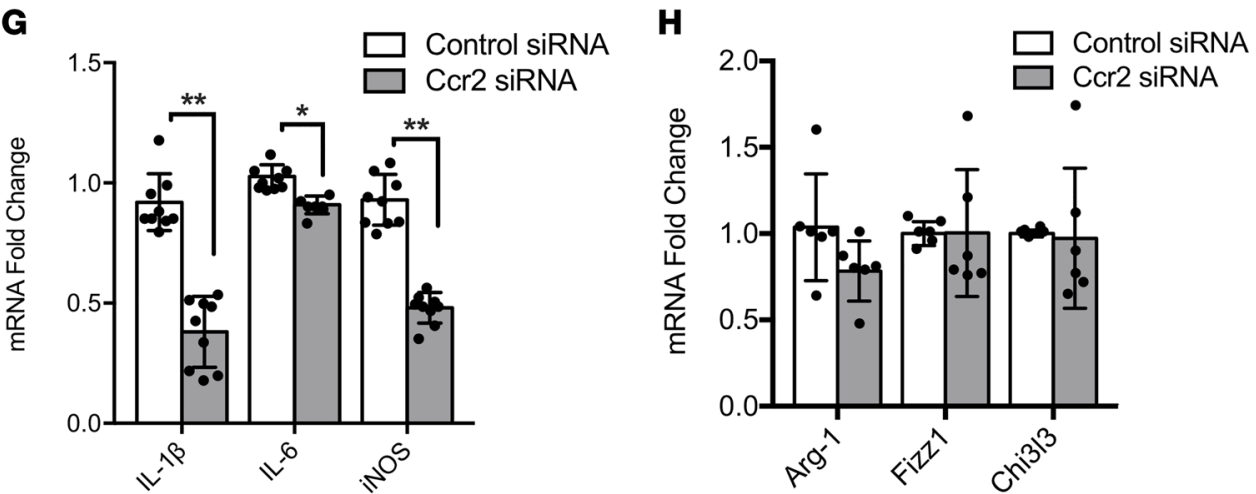

Figure 5. Ccl2 promotes M1 macrophage polarization through Ccr2. (A) Representative image of immunofluorescence staining with primary antibodies against iNOS or Arg-1. Nuclei were counterstained with 4'-6-diamidino-2-phenylindole dihydrochloride (DAPI). Original magnification x20. (B and C) Quantification of immunostaining for iNOS+ $(\mathbf{B})$ and $\mathrm{Arg}-1^{+}$(C) cells in BMDMs. Fluorescence-positive cells in 4 different high-power fields from each lung section were quantified using Image $\mathrm{v1.50}(\mathrm{NIH})$ and are presented as mean fluorescence intensity per square micrometer. $n=5$ high power view/group. ( $\boldsymbol{D}$ and E) Quantitative RT-PCR analyses for M1-associated genes (D) and M2-associated genes (E) in BMDMs with or without Ccl2 stimulation. $n=3 /$ group. (F) Quantitative RT-PCR analyses for Ccl2 expression in BMDMs with control siRNA and Ccr2 siRNA knockdown. $n=3 / g$ group. (G and $\mathbf{H}$ ) Quantitative

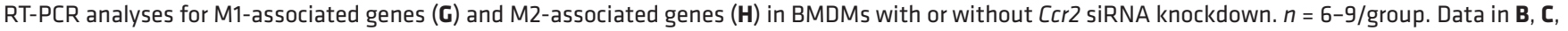
and $\mathbf{F}$ were compared by 2-way ANOVA. Data in $\mathbf{D}, \mathbf{G}$, and $\mathbf{H}$ were compared by 2-tailed Student's $t$ test. ${ }^{*} P<0.05 ;{ }^{* *} P<0.01 ;{ }^{* *} P<0.001$.

tified Ccl2 as one of the targets of miR-511-3p, and that activation of Ccl2 can enhance M1 macrophage polarization. Most importantly, we have identified RhoA signaling as a regulator of the Ccl2-induced macrophage polarization via Ccr2. Thus, our results advance our understanding of how the Mrcl-encoded miR511-3p contributes to Mrc1-mediated allergen-induced AHR and lung inflammation.

While studies on miR-511-3p are limited, several studies have suggested that it controls the activation of tumor-associated macrophages (19), regulates macrophage-mediated microbial responses (20), is involved in 
A

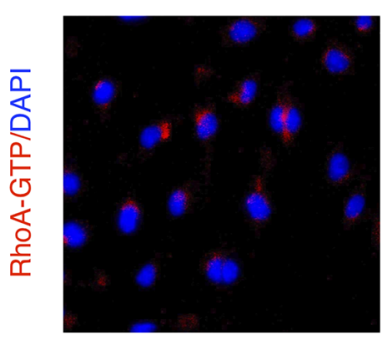

$\mathrm{Ccl} 2$

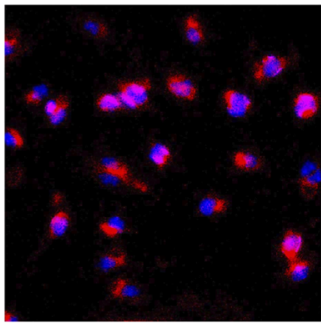

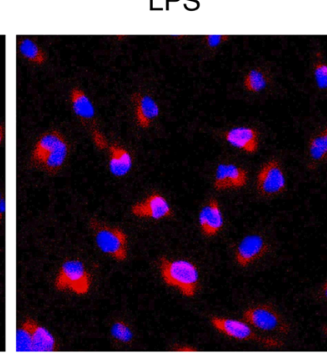

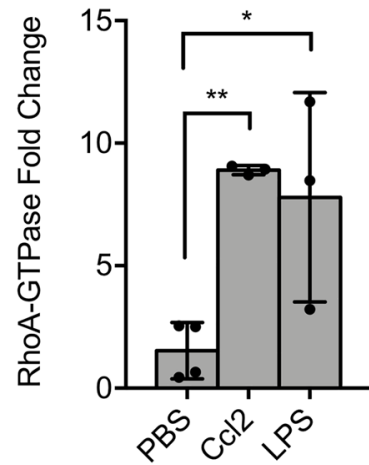

\section{B}

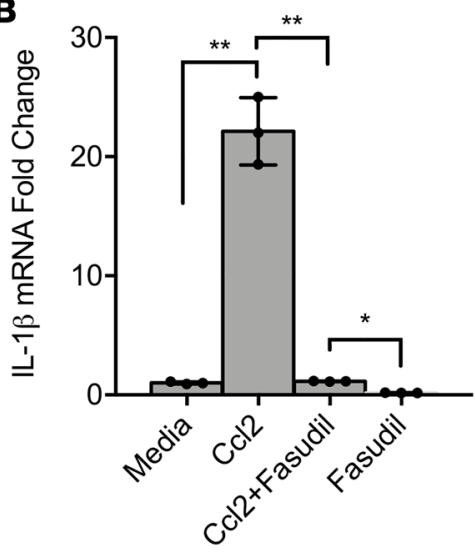

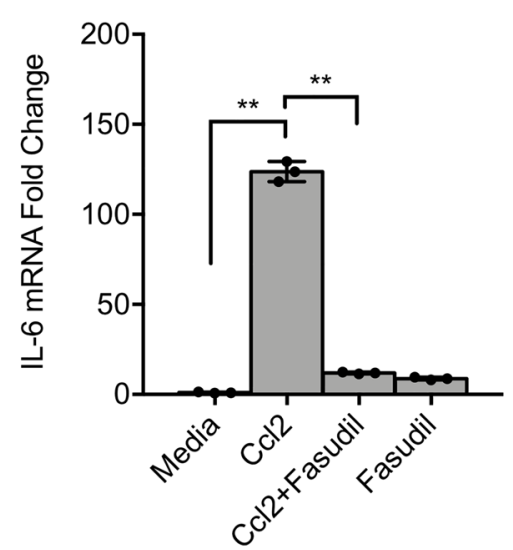

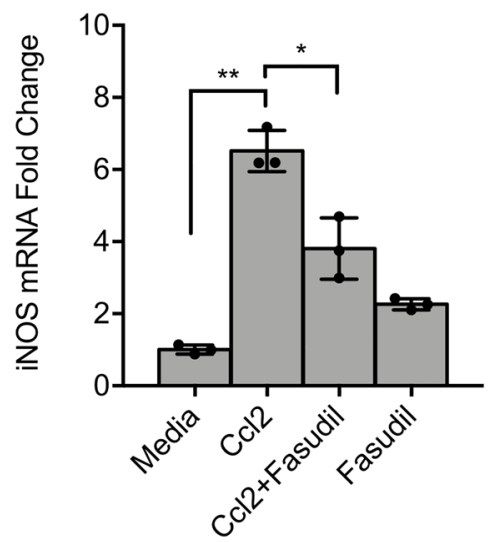

C

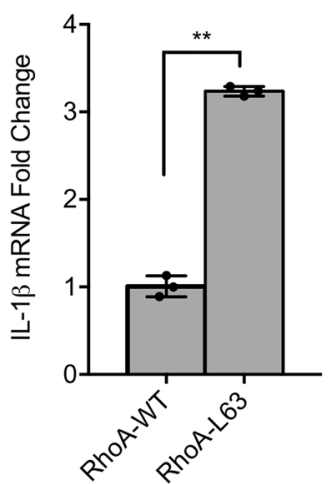

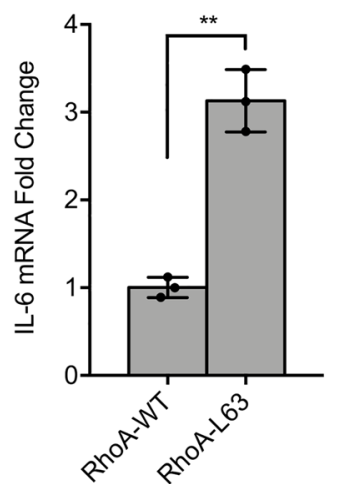
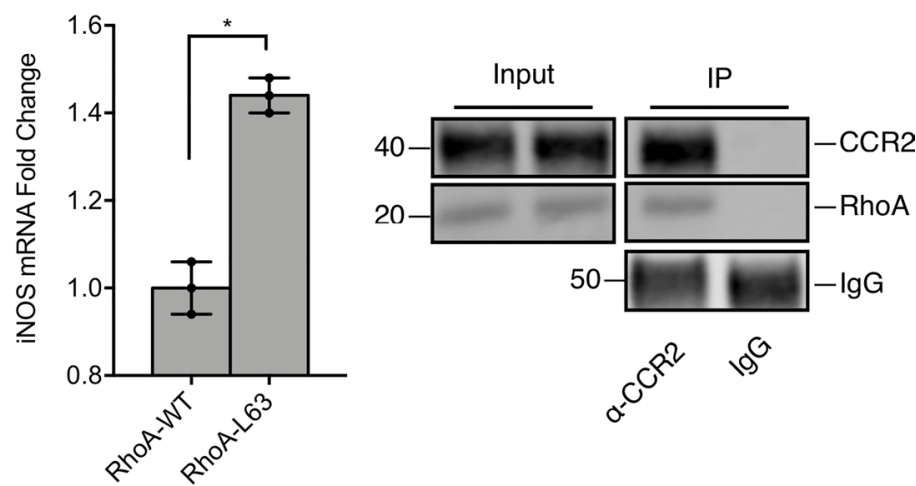

Figure 6. Ccl2 activates RhoA signaling that contributes to macrophage polarization. (A) Representative image of immunofluorescence staining with primary antibodies against RhoA-GTP in BMDMs. Nuclei were counterstained with 4'-6-diamidino-2-phenylindole dihydrochloride (DAPI). Right panel, quantification of RhoA-GTP+ cells ( $n=3-4$ /group). Original magnification x20. (B) Quantitative RT-PCR analysis for M1-associated genes in Ccl2-treated BMDMs with or without fasudil pretreatment ( $n=3$ /group). (C) Quantitative RT-PCR analysis for M1-associated genes in BMDMs treated with a plasmid carrying RhoA control (RhoA WT) and constitutively active RhoA (RhoA-L63) ( $n=3 /$ group). (D) Coimmunoprecipitation of CCR2 and RhoA in Ccl2-treated THP-1 cells. Data in A and B were compared by 2-way ANOVA. Data in C were compared by 2-tailed Student's $t$ test. ${ }^{*} P<0.05 ;{ }^{* *} P<0.01$.

the regulation of intestinal inflammation (20), and affects DC function by cross-talk with CLRs (21). Recent studies have also suggested that miR-511-3p may be a prognostic factor and potential tumor suppressor through inverse regulation of its downstream target gene AKT (serine/threonine-specific protein kinase 3) (34). Our studies provide further evidence that miR-511-3p may protect against allergen-induced airway inflammation (10). In particular, our studies raise the possibility that miR-511-3p is a major determinant in the increased inflammation observed in $\mathrm{Mrcl}^{1^{-1}}$ mice. First of all, miR-511-3p is mainly expressed in macrophages and DCs among all the different immune cells (Supplemental Figure 4), a pattern similar to that of Mrc1 that is mainly expressed in macrophages and DCs $(18,35)$. Thus, this finding further strengthens the notion that miR-511-3p is transcriptionally correlated with Mrc1 and plays an important role in Mrc1-mediated macrophage/DC functions or innate immune responses to environmental stimuli $(10,19)$. 
A

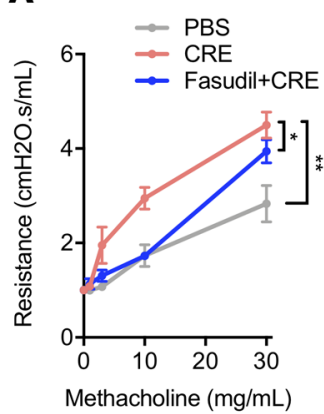

B
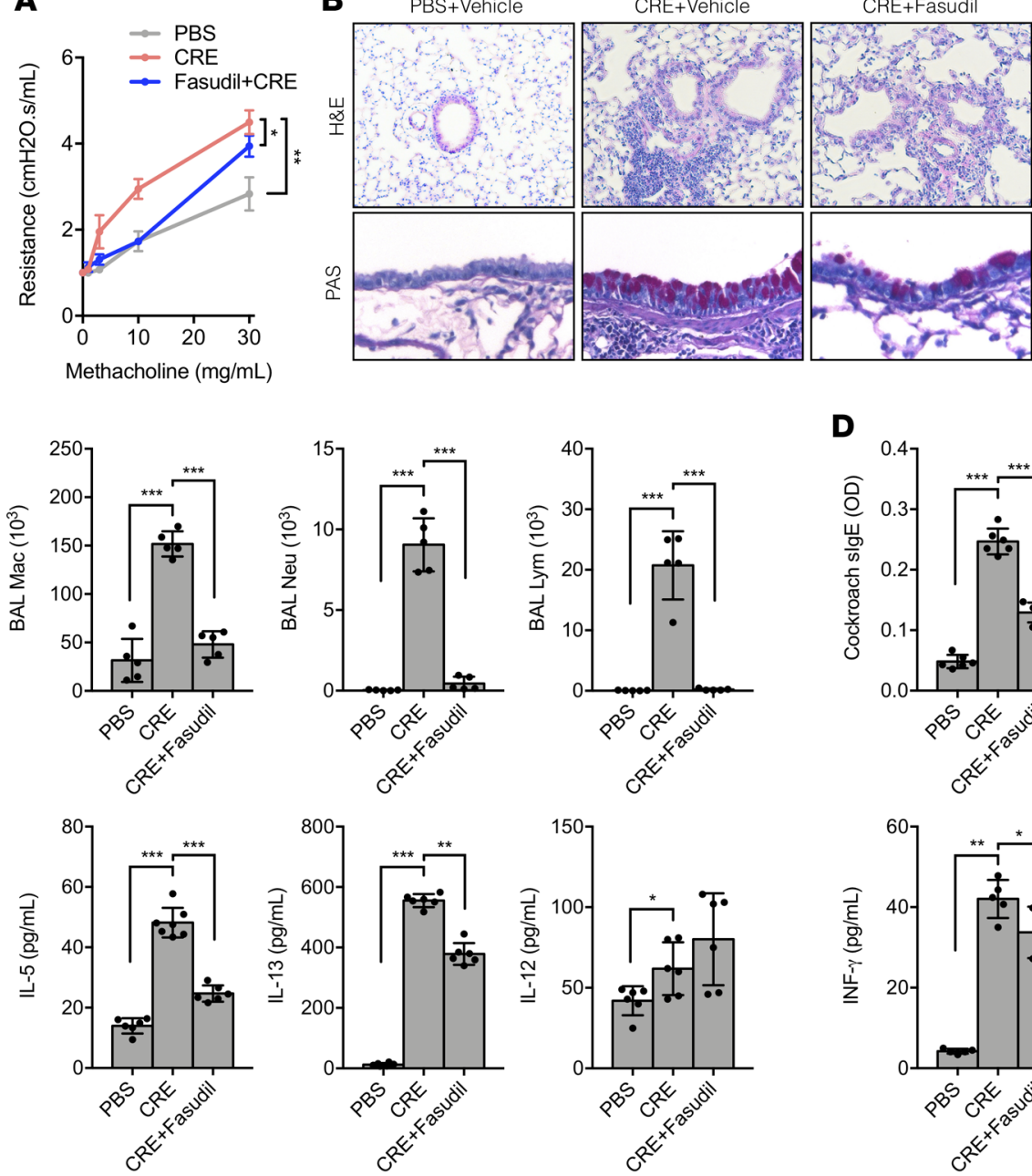

D
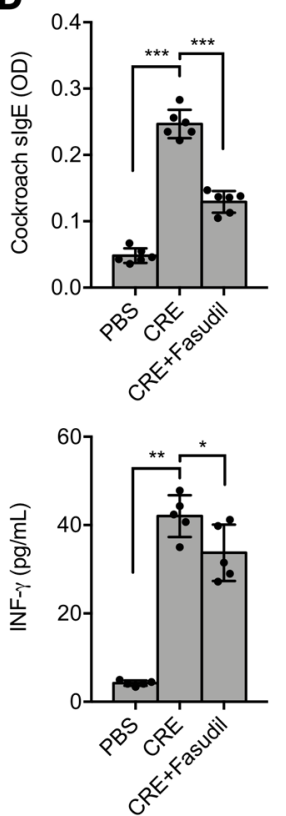
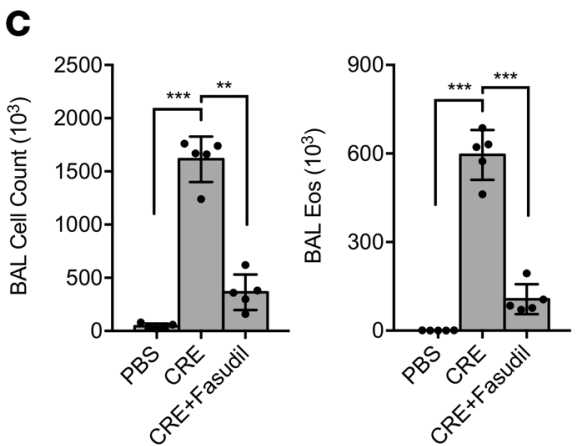

$\mathbf{E}$
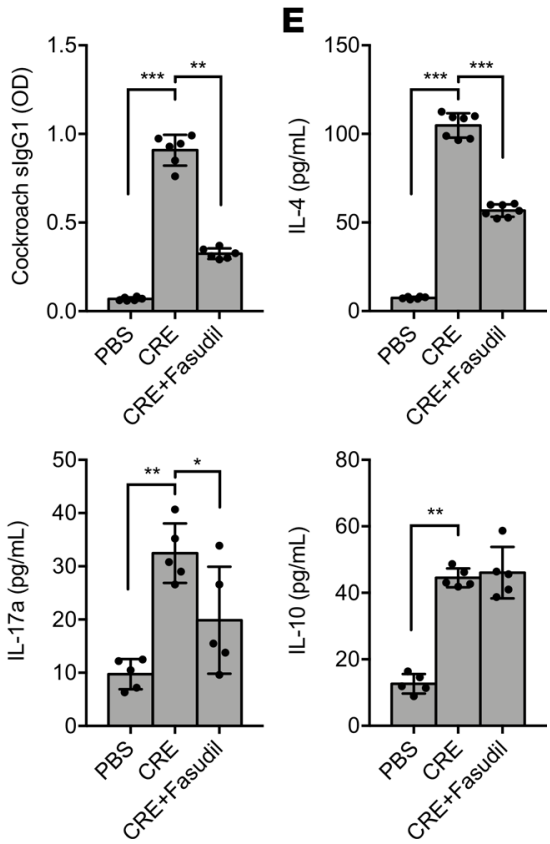

Figure 7. RhoA signaling regulates lung inflammation and macrophage polarization in a mouse model. (A) Lung resistance in response to increasing concentrations of methacholine using the forced oscillation technique (FlexiVent, SCIREQ) ( $n=4 /$ group). (B) Histological examination of mouse paraffin lung sections stained with hematoxylin and eosin (H\&E, upper panel) and periodic acid-Schiff (PAS, lower panel). Original magnification x10. (C) BAL fluid total and differential cell counts as assessed using flow cytometry ( $n=5 / g r o u p)$. (D) Serum levels of cockroach allergen-specific IgE and IgG1 ( $n=5$ /group). (E) BAL fluid cytokine levels ( $n=5-7 /$ group). Data in A and C-E were compared by 2-way ANOVA. ${ }^{*} P<0.05 ;{ }^{* *} P<0.01 ;{ }^{* * *} P<0.001$.

Second, when these $\mathrm{Mrcl}^{-1-}$ mice were transduced with miR-511-3p, the increased inflammation observed in $\mathrm{Mrcl}^{-/-}$mice was attenuated, indicating that miR-511-3p may play an important role in the allergen-induced lung inflammation. Third, we suggested the possible mechanism in which miR-511-3p prevents allergic lung inflammation through regulating macrophage polarization. Indeed, we found that AAV-miR-511-3p-transduced $\mathrm{Mrcl}^{-1-}$ mice showed macrophage polarization toward M2 in the lung tissue. It has been documented that M2 macrophages can protect against inflammation through the release of antiinflammatory cytokines (e.g., IL-10) (18). Although it is difficult to directly examine the production of cytokines by M1 or M2 macrophages in the allergen-challenged mouse model, we carefully characterized lung macrophages by not only coimmunostaining but also flow cytometry analysis with the markers suggested by Misharin et al. (36). Importantly, we used a similar approach and characterized macrophages enriched from BAL fluids of $\mathrm{Mrcl}^{-1-}$ mice treated with or without AAV-miR-511-3p. Furthermore, the expression of M1/M2-associated genes was also investigated in those macrophages. Consistently, macrophages in either lung tissues or BAL fluids from mice treated with miR-511-3p showed an increase in the M2 phenotype, indicating that miR511-3p polarizes macrophages toward the M2 phenotype that may contribute to the suppression of allergic lung inflammation. Additionally, we found that IL-10 in BAL fluids from mice treated with miR-511-3p was increased, suggesting that IL-10 may, at least partially, play a role in the protection against lung inflammation by miR-511-3p. However, no evidence was provided as to whether M2 macrophages are the major 


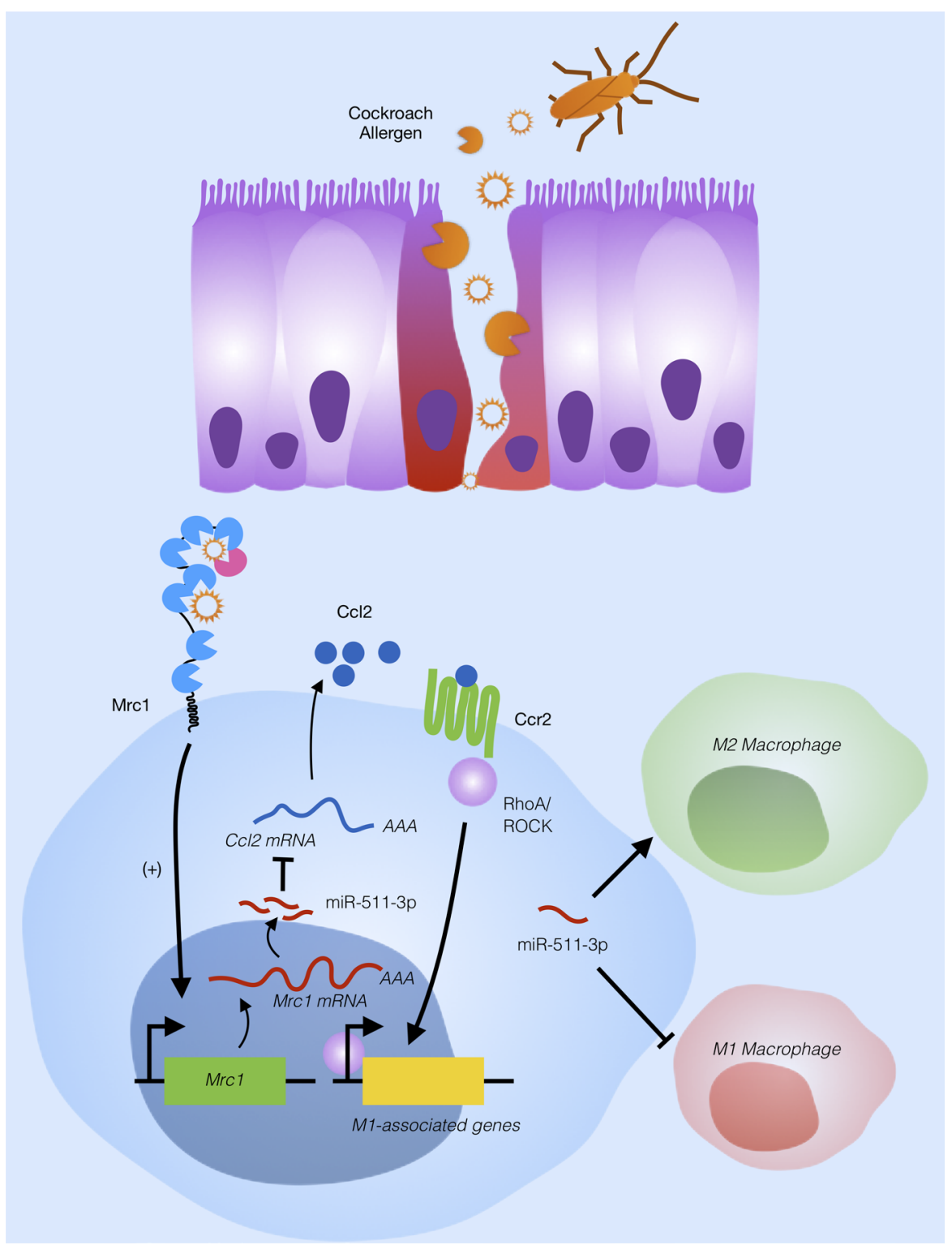

Figure 8. Schematic representation of findings. Exposure to cockroach allergen induces Mrc1 expression in macrophages for allergen uptake and the release of Mrc1-encoded miR-511-3p. The released miR-511-3p binds Ccl2 mRNA and inhibits the production of $\mathrm{Ccl} 2$. C $\mathrm{Cl} 2$ can polarize macrophages toward the M1 phenotype through its receptor $C \mathrm{cr} 2$ and subsequent activation of RhoA signaling. Thus, the insufficient amount of $\mathrm{Ccl} 2$ caused by miR-511-3p fails to polarize macrophages into an inflammatory M1 phenotype, thereby leading to the reduction of allergic inflammation.

source of IL-10 in the BAL fluids. Furthermore, we observed increased T regulatory cells (Tregs), one of the cellular sources of IL-10, in the lung tissues and BAL fluids of $\mathrm{Mrcl}^{-1-}$ mice treated with AAV-miR-511-3p (Supplemental Figure 5). Thus, future studies would be of interest to identify the underlying mechanisms by which M2 macrophages prevent allergic lung inflammation through the regulation of $\mathrm{T}$ cell differentiation and cytokine production. Additionally, since miR-511-3p is also expressed in DCs (33), it is very likely that miR-511-3p in DCs may contribute to the increased inflammation caused by the lack of Mrc1, which would also be of interest to determine in the future.

Next, we explored the fundamental mechanisms by which miR-511-3p confers protection against allergic inflammation. Several genes have been reported to be targeted by miR-511-3p, including $A K T$ (34), Rock2 (19, 37), Ltbp 1 (19), and Tlr4 (20). Among these, Rock2 and Ltbp1 were significantly downregulated, and Tlr4 was upregulated by miR-511-3p in our previous studies (10). Of interest, Rock2 is one of the downstream effector proteins of RhoA (38), and the activation of RhoA/ROCK signaling is responsible for the macrophage dif- 
ferentiation induced by PMA $(19,39)$. Furthermore, we have previously demonstrated that Ptgds (encoding hematopoietic prostaglandin D synthase), which catalyzes the conversion of PGH2 to PGD2, may be one of the direct target genes for miR-511-3p (10). Follow-up studies on the role of Ptgds and PGD2 in macrophage polarization and development of allergic asthma are ongoing in our laboratory. In this study, we performed a microarray analysis by using a different approach to identify miR-511-3p target genes that are highly expressed in M2 macrophages, but significantly antagonized by miR-511-3p. Several genes were identified, including the top-ranked genes $A k 2$ (25), Ccl2 (23), Clcn3 (26), Plek (27), and Srgn (28). These genes have been predicted to have miR-511-3p binding sites in their 3'-UTR. Thus far, no studies to our knowledge have reported these genes to be associated with allergic airway inflammation. Of these, $\mathrm{Ccl} 2$ belongs to the $\mathrm{C}-\mathrm{C}$ class of chemokines and has been shown to be a key modulator of inflammation, with specific chemotactic activity for monocytes/macrophages via preferential interaction with Ccr2 $(40,41)$. Alterations in the expression of CCL2 have been observed in BAL fluids from allergen-challenged asthmatic patients (24), and demonstrated its association with asthma (42-44). Thus, we prioritized $C c l 2$ as a potentially novel target gene for miR-511$3 p$ that may participate in miR-511-3p-regulated downstream immune responses in allergic asthma.

We provided several pieces of evidence to support the regulation of Ccl2 by miR-511-3p. First of all, we found by in vitro analysis that macrophages treated with miR-511-3p have reduced release of Ccl2. Second, BAL fluids from CRE-challenged mice contained higher levels of Ccl2, which were lower in those from miR-511-3p-treated mice. Third, we performed in silico analysis by using BiBiServ RNAhybrid and predicted the potential binding site for miR-511-3p in $C c l 2$ mRNA. The binding was further confirmed by the miR-mRNA pulldown assay that we developed by mixing biotinylated miR with cell lysates to identify miR binding partners $(33,45)$. Particularly, a significantly higher amount of $C c l 2 \mathrm{mRNA}$ was identified in miR511-3p-mRNA complexes that were isolated from macrophages, suggesting a possible physiological interaction between miR-511-3p and Cc12. Thus, these findings suggest a potentially novel epigenetic mechanism by which miR-511-3p may confer protection against allergic inflammation by inhibiting Cc12 expression.

A further mechanism elucidated by this study is that $\mathrm{Ccl} 2$ induces macrophage polarization to the M1 phenotype through its receptor, $\mathrm{Ccr} 2$. The $\mathrm{Cc} 12 / \mathrm{Ccr} 2$ pathway has been reported to skew macrophage polarization at the transcriptomic and functional level in human and mouse macrophages (46-49). Our studies here provide evidence that $\mathrm{Ccl} 2$ can induce $\mathrm{Ccr} 2$-dependent expression of M1-associated genes, but not M2-associated genes, and further suggested that the $\mathrm{Cc} 12 / \mathrm{Ccr} 2$ pathway may be associated with M1 macrophage polarization. Thus, the identification of the $\mathrm{Ccl} 2 / \mathrm{Ccr} 2$ pathway in controlling macrophage polarization may open new strategies for the treatment of allergic inflammation.

One of the most important mechanisms elucidated by our study is that active RhoA signaling plays a critical role in the $\mathrm{Ccl} 2 / \mathrm{Ccr} 2$ pathway-associated macrophage polarization. RhoA is an intracellular signal transducer of the Rho family of small GTPases (50). Ccl2 has been shown to induce RhoA activity that may contribute to cell migration, survival (51), and various other processes, such as the cell cycle, cytoskeletal regulation, and cellular growth $(52,53)$. RhoA signaling has been linked with pathophysiological processes of asthma, including the development of AHR, airway inflammation, and remodeling (54-61). Also, we found that asthmatic lung tissues showed activated RhoA signaling, and inhibition of RhoA signaling prevented CRE-induced airway inflammation and remodeling in a chronic mouse model of asthma (30). Furthermore, the activation of RhoA signaling has been associated with macrophage polarization $(62,63)$. In this study, we provided evidence that $\mathrm{Ccl} 2$ can activate RhoA signaling, and that inhibition of RhoA signaling can block Ccl2-induced M1 macrophage polarization. To further confirm the role of RhoA signaling in macrophage polarization, macrophages were transfected with a constitutively active RhoA. We found that macrophages with active RhoA expression tend to polarize toward M1 macrophages. Furthermore, our coimmunoprecipitation study suggested a possible physiological interaction between CCR2 and RhoA in macrophages. Taken together, these findings suggest that the $\mathrm{Ccl} 2 / \mathrm{CcR} 2$ axis may play a role in macrophage polarization through interacting with RhoA.

Recent studies have suggested that RhoA signaling plays a critical role in immune cell activation and migration, and has been considered as a key regulator of innate and adaptive immunity (64). In this study, we used fasudil, a potent ROCK inhibitor, to inhibit RhoA signaling in vivo and examine the effect of RhoA signaling in allergic lung inflammation. We found that fasudil could inhibit CRE-induced airway resistance and lung inflammation. By using the same approach as that described above, we characterized the lung macrophages and found that mice treated with fasudil showed decreased M1, but increased M2 macrophages (data not shown). These results raise the possibility that RhoA signaling regulates macrophage polarization that may contribute to CRE-induced lung inflammation. Given that studies have sug- 
gested that inhibition of RhoA signaling can regulate $\mathrm{T}$ cell differentiation (58) and suppress the production of Th17 cytokines (e.g., IL-21, IL-17) $(65,66)$, we expect that RhoA signaling may play a critical role in allergic inflammation through the regulation of $\mathrm{T}$ cell differentiation and cytokine production. Although it remains unknown whether RhoA signaling regulates DC function, it would be critical to investigate the effect of RhoA signaling in miR-511-3p/Cc12/Ccr2-mediated DC differentiation, antigen presentation, and $\mathrm{T}$ cell differentiation in allergic asthma in the future.

In summary, we have determined the functional significance of miR-511-3p in Mrc1-mediated allergen-induced lung inflammation, and explored the underlying mechanisms by which miR-511-3p attenuates allergic inflammation and promotes M2 macrophage polarization. Although other identified target genes may be critical, e.g., $A K T$ (34), Rock2 (19, 37), Ltbp1 (19), and Ptgds (10), our data provide evidence that Ccl2 is one of the target genes for miR-511-3p and suggest a potentially novel mechanism by which Ccl2 induces macrophage polarization through the Ccr2/RhoA axis (Figure 8). Thus, our data allow us to conclude that targeting miR-511-3p and its target genes may yield therapeutic approaches for the treatment of allergic asthma.

\section{Methods}

Mice. Mrc1-deficient (Mrcl-1-) and C57BL/6J (WT) mice were purchased from the Jackson Laboratory and maintained under specific pathogen-free conditions at the animal facility of the Johns Hopkins University School of Medicine. Mice were at 6-8 weeks of age and in all experiments, all mice were age and sex matched.

$M r c 1^{-1-}$ mice transfected with $A A V-m i R-511-3 p$. AAV, a nonpathogenic small DNA virus, has been utilized to deliver DNA to target cells (67). An AAV serotype 9 (AAV9) vector encoding the enhanced GFP reporter and expressing miR-511-3p (AAV-CMV-miR-511-3p-GFP), driven by the cytomegalovirus (CMV) promoter, was generated in SignaGen Laboratory. The methods for the transfection of AAV into $\mathrm{Mrc1}^{-/-}$mice and detection of GFP expression were previously described (10). AAV at a dose of $5 \times 10^{10} \mathrm{PFU}$ in $20 \mu \mathrm{L}$ $\mathrm{PBS} /$ mouse was administered to mice via intratracheal injection. GFP expression in the lung tissues was detected on day 35 after AAV injection.

CRE-induced mouse model of asthma and the analysis of lung inflammation. Generation of a CRE-induced mouse model of asthma and analysis of lung inflammation was established as previously described (10, 33). In a separate experiment, mice were treated with fasudil (Selleckchem) at a dose of $30 \mathrm{mg} / \mathrm{kg}$ dissolved in water by intratracheal administration 30 minutes prior to CRE (Greer Laboratory) challenge. Vehicle-treated mice received PBS.

Measurement of AHR. Mice were anesthetized with a mixture of $90 \mathrm{mg} / \mathrm{kg}$ ketamine and $18 \mathrm{mg} / \mathrm{kg}$ xylazine, and a tracheotomy tube was inserted. Ventilation was initiated with a volume-cycled ventilator (Flexivent, SCIREQ Scientific) with a positive-end expiratory pressure of $2 \mathrm{cmH}_{2} \mathrm{O}$. Airway responsiveness was measured by challenging mice to increasing doses of aerosolized methacholine $(0-30 \mathrm{mg} / \mathrm{mL})$. The airway resistance was measured with the Flexivent software and exported to Pulmodyn data-acquisition software (Hugo Sachs Electronic) for data analysis (68).

ELISA. Concentrations of IL-4, IL-5, IL-13, IL-12, IL-17, IFN- $\gamma$, IL-10, and Cc12 in cell-free BAL fluids were measured by using the Ready-Set-Go! ELISA sets (Thermo Fisher Scientific) (10, 33, 68). CRE-specific sIgE and sIgG1 serum levels were analyzed by ELISA, as previously described $(10,33,68)$.

Immunofluorescence staining. Immunofluorescence staining was performed as previously reported (10, 68, 69). The following antibodies were used: F4/80 (BM8, Thermo Fisher Scientific), Arg-1 (D4E3M, Cell Signaling Technology), iNOS (D6B6S, Cell Signaling Technology), and RhoA-GTPase (26904, New East Biosciences). Detailed information is provided in Supplemental Table 2. To determine the fluorescence signal in tissue sections, fluorescence-positive cells in 4 different high-power fields from each lung section were quantified using ImageJ v1.50e (NIH) and presented as mean fluorescence intensity per square micrometer. Four to 6 lung sections from each sample were used for analysis.

Flow cytometry analysis. Single-cell suspensions from whole lung tissues were prepared from minced lung tissues as described in the supplementary materials. To characterize the M1/M2 macrophages, the resuspended cells were stained with antibodies against F4/80 (BM8, BioLegend), CD11c (HL3, BD), Arg1 (D4E3M, Cell Signaling Technology), or iNOS (NB300-605, Novus). Macrophages enriched from the BAL fluids were stained by using the same methods. To characterize Tregs, the cells were first stained with antibodies against CD4 (RM4-5, BioLegend) and CD25 (PC61.5, BioLegend), followed by intracellular staining with FoxP3 antibody (FJK-16s, Thermo Fisher Scientific). These stained samples were then analyzed on a FACSCalibur flow cytometer (BD Biosciences). 
RT-PCR. Quantitative RT-PCR was performed as previously reported $(10,68,69)$. Primer sequences are listed in Supplemental Table 3.

miR-511-3p overexpression in macrophages with LV transduction. BMDMs were generated and then transduced with LV-miR-511-3p or a mutated miR-511-3p (LV-Mock) as previously described (9). Both LVmiR-511-3p and LV-Mock were provided by Mario Leonardo Squadrito (Swiss Federal Institute of Technology Lausanne [EPFL], Lausanne, Switzerland).

Transfection of constitutively activate RhoA into macrophages. Approximately $1.0 \times 10^{5} \mathrm{BMDMs}$ were seeded onto a 6-well tissue culture plate 24 hours prior to transfection with a plasmid expressing a constitutively active RhoA (myc-RhoA-Q63L-GFP) or empty vector (EV-GFP) control using FuGENE 6 (Promega) as instructed by the manufacturer (70). Transfection efficiency was assessed by GFP expression using flow cytometry.

Macrophage polarization. Macrophages were polarized with either IL-4 (20 ng/mL) or LPS (100 ng/mL) for 24 or 6 hours, respectively, and then analyzed for M1 and M2 macrophage markers (10). For Ccl2-induced macrophage polarization, BMDMs were treated with recombinant murine Ccl2 at 5, 20, or $50 \mathrm{ng} /$ $\mathrm{mL}$ (BioLegend). For some experiments, BMDMs were treated with fasudil with or without Ccl2. Macrophage polarization was assessed by immunostaining and/or qRT-PCR.

Transfection of miR mimics and siRNA. miR-511-3p mimic and its corresponding negative control were purchased from Thermo Fisher Scientific. Ccr2 knockdown was accomplished by using a predesigned MISSION siRNA pair (Sigma-Aldrich). miR and siRNA transfection were performed using Lipofectamine RNAiMAX (Thermo Fisher Scientific) as instructed by the manufacturer.

Coimmunoprecipitation. Coimmunoprecipitation was performed as described in the supplementary materials (70). RhoA expression in THP-1 cells was enriched with $50 \mathrm{ng} / \mathrm{mL}$ of recombinant human CCL2 (BioLegend) for 2 hours. These cells were lysed and then immunoprecipitated using specific antibody against CCR2 (Cell Signaling Technology, D14H7) and isotype control (Cell Signaling Technology, DA1E), respectively. The antibody/protein complex was enriched using Protein A magnetic beads followed by Western blot using an antibody against RhoA.

Analysis of miR-mRNA interaction. The analysis of miR-mRNA interaction was performed as previously described (33). Briefly, a synthetic miR-511-3p containing a biotin at the 3 'end was annealed with a complementary strand (Integrated DNA Technologies). Twenty nanograms of the duplex was incubated with BMDM cell lysates, and miR-mRNA complexes were isolated using streptavidin beads. These isolated complexes were then reverse transcribed using the High-Capacity Reverse Transcription Kit (Thermo Fisher Scientific). qPCR was performed by using Ccl2 primers to identify bound mRNA, and the enrichment was analyzed by the foldchange in miR-511-3p pulldown versus that of the negative control miR (cel-miR-39 biotinylated miR).

Microarray analysis. Microarray analysis of mRNA expression in BMDMs untreated, treated with IL-4 or IL-4 and miR-511-3p mimic, was performed by using MouseRef- 8 vs Expression ReadChip arrays (Illumina). Microarray assay and data analysis were performed as previously described (10). MIAME-compliant gene expression array data have been deposited to the NCBI's Gene Expression Omnibus (GEO GSE137120) (71).

Statistical analysis. All data are expressed as the mean \pm SEM for each group. Statistical significance for normally distributed samples was assessed by an independent 2-tailed Student's $t$ test or 2-way ANOVA test. Analyses were performed with GraphPad Prism version 5.1 software. $P<0.05$ was considered statistically significant for all analyses.

Study approval. The experimental procedures involving the use and care of animals were approved by the Animal Care and Use Committee at Johns Hopkins University School of Medicine, Baltimore, Maryland.

\section{Author contributions}

DCD, JM, XK, KS, and ZQ performed experiments and analyzed data. DCD, and PG wrote the manuscript. PG, MW, and FTI designed and supervised the study. All authors read and approved the final manuscript.

\section{Acknowledgments}

This work was supported by grants from the US NIH (R01 ES021739, R21 AI109062, R21 AI121768, and R01 AI141642).

Address correspondence to: Peisong Gao, Johns Hopkins Asthma and Allergy Center, 5501 Hopkins Bayview Circle, Room 3B.71, Baltimore, Maryland 21224, USA. Phone: 410.550.2124; Email: pgao1@jhmi.edu. 
1. Tsai YM, et al. Functional interaction of cockroach allergens and mannose receptor (CD206) in human circulating fibrocytes. PLoS One. 2013;8(5):e64105.

2. Emara M, et al. Recognition of the major cat allergen Fel d 1 through the cysteine-rich domain of the mannose receptor determines its allergenicity. J Biol Chem. 2011;286(15):13033-13040.

3. Meyer-Wentrup F, et al. DCIR is endocytosed into human dendritic cells and inhibits TLR8-mediated cytokine production. J Leukoc Biol. 2009;85(3):518-525.

4. Santiago L, Daniels G, Wang D, Deng FM, Lee P. Wnt signaling pathway protein LEF1 in cancer, as a biomarker for prognosis and a target for treatment. Am J Cancer Res. 2017;7(6):1389-1406.

5. Shreffler WG, et al. The major glycoprotein allergen from Arachis hypogaea, Ara h 1, is a ligand of dendritic cell-specific ICAM-grabbing nonintegrin and acts as a Th2 adjuvant in vitro. J Immunol. 2006;177(6):3677-3685.

6. Do DC, Zhao Y, Gao P. Cockroach allergen exposure and risk of asthma. Allergy. 2016;71(4):463-474.

7. Hadebe S, Brombacher F, Brown GD. C-type lectin receptors in asthma. Front Immunol. 2018;9:733

8. Do DC, Yang S, Yao X, Hamilton RG, Schroeder JT, Gao P. N-glycan in cockroach allergen regulates human basophil function. Immun Inflamm Dis. 2017;5(4):386-399.

9. Royer PJ, et al. The mannose receptor mediates the uptake of diverse native allergens by dendritic cells and determines allergen-induced T cell polarization through modulation of IDO activity. J Immunol. 2010;185(3):1522-1531.

10. Zhou Y, et al. Mannose receptor modulates macrophage polarization and allergic inflammation through miR-511-3p. J Allergy Clin Immunol. 2018;141(1):350-364.e8.

11. Sorvillo N, et al. The macrophage mannose receptor promotes uptake of ADAMTS13 by dendritic cells. Blood. 2012;119(16):3828-3835.

12. Shukla GC, Singh J, Barik S. MicroRNAs: processing, maturation, target recognition and regulatory functions. Mol Cell Pharmacol. 2011;3(3):83-92.

13. Simpson LJ, et al. A microRNA upregulated in asthma airway T cells promotes TH2 cytokine production. Nat Immunol. 2014;15(12):1162-1170.

14. Mattes J, Collison A, Plank M, Phipps S, Foster PS. Antagonism of microRNA-126 suppresses the effector function of TH2 cells and the development of allergic airways disease. Proc Natl Acad Sci USA. 2009;106(44):18704-18709.

15. Bazzoni F, et al. Induction and regulatory function of miR-9 in human monocytes and neutrophils exposed to proinflammatory signals. Proc Natl Acad Sci USA. 2009;106(13):5282-5287.

16. Self-Fordham JB, Naqvi AR, Uttamani JR, Kulkarni V, Nares S. MicroRNA: dynamic regulators of macrophage polarization and plasticity. Front Immunol. 2017;8:1062.

17. Li J, et al. Critical role of alternative M2 skewing in miR-155 deletion-mediated protection of colitis. Front Immunol. 2018;9:904

18. Saradna A, Do DC, Kumar S, Fu QL, Gao P. Macrophage polarization and allergic asthma. Transl Res. 2018;191:1-14.

19. Squadrito ML, et al. miR-511-3p modulates genetic programs of tumor-associated macrophages. Cell Rep. 2012;1(2):141-154.

20. Heinsbroek SE, et al. miR-511-3p, embedded in the macrophage mannose receptor gene, contributes to intestinal inflammation. Mucosal Immunol. 2016;9(4):960-973.

21. Awuah D, Alobaid M, Latif A, Salazar F, Emes RD, Ghaemmaghami AM. The cross-talk between miR-511-3p and C-type lectin receptors on dendritic cells affects dendritic cell function. J Immunol. 2019;203(1):148-157.

22. Karo-Atar D, Itan M, Pasmanik-Chor M, Munitz A. MicroRNA profiling reveals opposing expression patterns for miR-511 in alternatively and classically activated macrophages. J Asthma. 2015;52(6):545-553.

23. Castela M, Nassar D, Sbeih M, Jachiet M, Wang Z, Aractingi S. Ccl2/Ccr2 signalling recruits a distinct fetal microchimeric population that rescues delayed maternal wound healing. Nat Commun. 2017;8:15463.

24. Lee YG, et al. Recruited alveolar macrophages, in response to airway epithelial-derived monocyte chemoattractant protein 1/ $\mathrm{CCl}$, regulate airway inflammation and remodeling in allergic asthma. Am J Respir Cell Mol Biol. 2015;52(6):772-784.

25. Rissone A, et al. Reticular dysgenesis-associated AK2 protects hematopoietic stem and progenitor cell development from oxidative stress. JExp Med. 2015;212(8):1185-1202.

26. Lamb FS, Graeff RW, Clayton GH, Smith RL, Schutte BC, McCray PB. Ontogeny of CLCN3 chloride channel gene expression in human pulmonary epithelium. Am J Respir Cell Mol Biol. 2001;24(4):376-381.

27. Cmarik JL, Hegamyer G, Gerrard B, Dean M, Colburn NH. cDNA cloning and mapping of mouse pleckstrin (Plek), a gene upregulated in transformation-resistant cells. Genomics. 2000;66(2):204-212.

28. Guo JY, et al. Serglycin in tumor microenvironment promotes non-small cell lung cancer aggressiveness in a CD44-dependent manner. Oncogene. 2017;36(17):2457-2471.

29. Rehmsmeier M, Steffen P, Hochsmann M, Giegerich R. Fast and effective prediction of microRNA/target duplexes. RNA. 2004;10(10):1507-1517.

30. Ke X, et al. Ras homolog family member A/Rho-associated protein kinase 1 signaling modulates lineage commitment of mesenchymal stem cells in asthmatic patients through lymphoid enhancer-binding factor 1. J Allergy Clin Immunol. 2019;143(4):1560-1574.e6.

31. Nagumo H, Sasaki Y, Ono Y, Okamoto H, Seto M, Takuwa Y. Rho kinase inhibitor HA-1077 prevents Rho-mediated myosin phosphatase inhibition in smooth muscle cells. Am J Physiol, Cell Physiol. 2000;278(1):C57-C65.

32. Tsuchiya S, Yamabe M, Yamaguchi Y, Kobayashi Y, Konno T, Tada K. Establishment and characterization of a human acute monocytic leukemia cell line (THP-1). Int J Cancer. 1980;26(2):171-176.

33. Qiu L, et al. miR-155 modulates cockroach allergen- and oxidative stress-induced cyclooxygenase-2 in asthma. J Immunol. 2018;201(3):916-929.

34. Zhang F, Wu Z. Significantly altered expression of miR-511-3p and its target AKT3 has negative prognostic value in human prostate cancer. Biochimie. 2017;140:66-72.

35. Salazar F, et al. The mannose receptor negatively modulates the Toll-like receptor 4-aryl hydrocarbon receptor-indoleamine 2,3-dioxygenase axis in dendritic cells affecting T helper cell polarization. J Allergy Clin Immunol. 2016;137(6):1841-1851.e2.

36. Misharin AV, Morales-Nebreda L, Mutlu GM, Budinger GR, Perlman H. Flow cytometric analysis of macrophages and dendritic cell subsets in the mouse lung. Am J Respir Cell Mol Biol. 2013;49(4):503-510. 
37. Zandi S, et al. ROCK-isoform-specific polarization of macrophages associated with age-related macular degeneration. Cell Rep . 2015;10(7):1173-1186.

38. Bishop AL, Hall A. Rho GTPases and their effector proteins. Biochem J. 2000;348 Pt 2:241-255.

39. Yang L, Dai F, Tang L, Le Y, Yao W. Macrophage differentiation induced by PMA is mediated by activation of RhoA/ROCK signaling. J Toxicol Sci. 2017;42(6):763-771.

40. Serbina NV, Jia T, Hohl TM, Pamer EG. Monocyte-mediated defense against microbial pathogens. Annu Rev Immunol. 2008;26:421-452.

41. Deshmane SL, Kremlev S, Amini S, Sawaya BE. Monocyte chemoattractant protein-1 (MCP-1): an overview. J Interferon Cytokine Res. 2009;29(6):313-326.

42. Yao TC, et al. MCP-1 gene regulatory region polymorphism in Chinese children with mild, moderate and near-fatal asthma. Allergy. 2004;59(4):436-441.

43. Chelbi H, et al. A polymorphism in the CCL2 chemokine gene is associated with asthma risk: a case-control and a family study in Tunisia. Genes Immun. 2008;9(7):575-581.

44. Yang EJ, Choi E, Ko J, Kim DH, Lee JS, Kim IS. Differential effect of CCL2 on constitutive neutrophil apoptosis between normal and asthmatic subjects. J Cell Physiol. 2012;227(6):2567-2577.

45. Panganiban RP, Pinkerton MH, Maru SY, Jefferson SJ, Roff AN, Ishmael FT. Differential microRNA epression in asthma and the role of miR-1248 in regulation of IL-5. Am J Clin Exp Immunol. 2012;1(2):154-165.

46. Sierra-Filardi E, et al. CCL2 shapes macrophage polarization by GM-CSF and M-CSF: identification of CCL2/CCR2-dependent gene expression profile. J Immunol. 2014;192(8):3858-3867.

47. Deci MB, Ferguson SW, Scatigno SL, Nguyen J. Modulating macrophage polarization through CCR2 inhibition and multivalent engagement. Mol Pharm. 2018;15(7):2721-2731.

48. He M, et al. Astrocyte-derived CCL2 is associated with M1 activation and recruitment of cultured microglial cells. Cell Physiol Biochem. 2016;38(3):859-870.

49. Zhang Y, et al. Kif4A mediate the accumulation and reeducation of THP-1 derived macrophages via regulation of CCL2-CCR2 expression in crosstalking with OSCC. Sci Rep. 2017;7(1):2226.

50. Etienne-Manneville S, Hall A. Rho GTPases in cell biology. Nature. 2002;420(6916):629-635.

51. Fang WB, Jokar I, Zou A, Lambert D, Dendukuri P, Cheng N. CCL2/CCR2 chemokine signaling coordinates survival and motility of breast cancer cells through Smad3 protein- and p42/44 mitogen-activated protein kinase (MAPK)-dependent mechanisms. J Biol Chem. 2012;287(43):36593-36608.

52. Burridge K, Wennerberg K. Rho and Rac take center stage. Cell. 2004;116(2):167-179.

53. Sahai E, Marshall CJ. RHO-GTPases and cancer. Nat Rev Cancer. 2002;2(2):133-142.

54. Takeda N, Kondo M, Ito S, Ito Y, Shimokata K, Kume H. Role of RhoA inactivation in reduced cell proliferation of human airway smooth muscle by simvastatin. Am J Respir Cell Mol Biol. 2006;35(6):722-729.

55. Kume H. RhoA/Rho-kinase as a therapeutic target in asthma. Curr Med Chem. 2008;15(27):2876-2885.

56. Goto K, Chiba Y, Sakai H, Misawa M. Mechanism of inhibitory effect of prednisolone on RhoA upregulation in human bronchial smooth muscle cells. Biol Pharm Bull. 2010;33(4):710-713.

57. Wei B, Shang YX, Li M, Jiang J, Zhang H. Cytoskeleton changes of airway smooth muscle cells in juvenile rats with airway remodeling in asthma and the RhoA/ROCK signaling pathway mechanism. Genet Mol Res. 2014;13(1):559-569.

58. Yang JQ, et al. RhoA orchestrates glycolysis for TH2 cell differentiation and allergic airway inflammation. J Allergy Clin Immunol. 2016;137(1):231-245.e4.

59. Desai LP, Aryal AM, Ceacareanu B, Hassid A, Waters CM. RhoA and Rac1 are both required for efficient wound closure of airway epithelial cells. Am J Physiol Lung Cell Mol Physiol. 2004;287(6):L1134-L1144.

60. Zhou Y, et al. Inhibition of mechanosensitive signaling in myofibroblasts ameliorates experimental pulmonary fibrosis. J Clin Invest. 2013;123(3):1096-1108

61. Knipe RS, et al. The Rho kinase isoforms ROCK1 and ROCK2 each contribute to the development of experimental pulmonary fibrosis. Am J Respir Cell Mol Biol. 2018;58(4):471-481.

62. Yang L, Dai F, Tang L, Le Y, Yao W. Macrophage differentiation induced by PMA is mediated by activation of RhoA/ROCK signaling. J Toxicol Sci. 2017;42(6):763-771.

63. Tian L, et al. Cannabinoid receptor 1 participates in liver inflammation by promoting M1 macrophage polarization via RhoA/ NF-кB p65 and ERK1/2 pathways, respectively, in mouse liver fibrogenesis. Front Immunol. 2017;8:1214.

64. Bros M, Haas K, Moll L, Grabbe S. RhoA as a key regulator of innate and adaptive immunity. Cells. 2019;8(7):E733.

65. Biswas SK, Mantovani A. Macrophage plasticity and interaction with lymphocyte subsets: cancer as a paradigm. Nat Immunol. 2010;11(10):889-896.

66. Zanin-Zhorov A, et al. Selective oral ROCK2 inhibitor down-regulates IL-21 and IL-17 secretion in human T cells via STAT3-dependent mechanism. Proc Natl Acad Sci USA. 2014;111(47):16814-16819.

67. Yang YS, et al. Bone-targeting AAV-mediated silencing of Schnurri-3 prevents bone loss in osteoporosis. Nat Commun. 2019;10(1):2958.

68. Qu J, et al. Oxidized CaMKII promotes asthma through the activation of mast cells. JCI Insight. 2017;2(1):e90139.

69. Wang $\mathrm{H}$, et al. Functional role of kynurenine and aryl hydrocarbon receptor axis in chronic rhinosinusitis with nasal polyps. $J$ Allergy Clin Immunol. 2018;141(2):586-600.e6.

70. Li C, et al. RhoA determines lineage fate of mesenchymal stem cells by modulating CTGF-VEGF complex in extracellular matrix. Nat Commun. 2016;7:11455.

71. Edgar R, Domrachev M, Lash AE. Gene Expression Omnibus: NCBI gene expression and hybridization array data repository. Nucleic Acids Res. 2002;30(1):207-210. 